\title{
Packing subgroups in relatively hyperbolic groups
}

\author{
G Christopher HrusKa \\ DANIEL T WISE
}

\begin{abstract}
We introduce the bounded packing property for a subgroup of a countable discrete group $G$. This property gives a finite upper bound on the number of left cosets of the subgroup that are pairwise close in $G$. We establish basic properties of bounded packing and give many examples; for instance, every subgroup of a countable, virtually nilpotent group has bounded packing. We explain several natural connections between bounded packing and group actions on $\mathrm{CAT}(0)$ cube complexes.

Our main result establishes the bounded packing of relatively quasiconvex subgroups of a relatively hyperbolic group, under mild hypotheses. As an application, we prove that relatively quasiconvex subgroups have finite height and width, properties that strongly restrict the way families of distinct conjugates of the subgroup can intersect. We prove that an infinite, nonparabolic relatively quasiconvex subgroup of a relatively hyperbolic group has finite index in its commensurator. We also prove a virtual malnormality theorem for separable, relatively quasiconvex subgroups, which is new even in the word hyperbolic case.
\end{abstract}

20F65; 20F67, 20F69

\section{Introduction}

In treating groups as geometric objects, it is natural to emphasize certain subgroups for special treatment, and these "quasiconvex" subgroups play a role akin to convex subspaces of a geodesic metric space. While for arbitrary groups the quasiconvexity of a subgroup is not even well-defined, for word-hyperbolic groups quasiconvexity is independent of the choice of generating system, and there is a useful theory of such subgroups which endows them with the properties of the ambient group, and then examines the favorable way in which they are embedded.

There has been substantial progress in the past few years generalizing properties of wordhyperbolic groups to properties of relatively hyperbolic groups with hypotheses on the peripheral subgroups. This paper is part of this trend where we focus on generalizations of ideas related to a certain important property of a quasiconvex subgroup. Let us first discuss the background of this property before indicating our generalizations. 
An infinite quasiconvex subgroup $H$ of a word-hyperbolic group $G$ cannot be normal unless it is of finite index (see Short [1]). More generally, there are substantial limits on the way it intersects its conjugates. The definitive result in this direction, obtained by Gitik-Mitra-Rips-Sageev [8], states that a quasiconvex subgroup has "finite width". This roughly means that there is an upper bound on the number of conjugates whose pairwise intersection is infinite. More precisely the width of $H$ in $G$ equals $(n-1)$ if $n$ is the smallest number with the property that for any $n$ distinct right cosets $g_{1} H, \ldots, g_{n} H$ the intersection $g_{i} H g_{i}^{-1} \cap g_{j} H g_{j}^{-1}$ is finite for some $i, j$. It is unknown whether having finite width characterizes quasiconvexity, but it seems unlikely that one could jump from an algebraic hypothesis to a geometric conclusion in this context.

\subsection{Bounded packing}

The geometric mechanism lying behind the finite width conclusion is what we call "bounded packing." In a metric space $(X, d)$ the distance between two subsets $Y$ and $Z$ is the infimum of distances between points $y \in Y$ and $z \in Z$. If $H$ is a subgroup of $G$, then $H$ has bounded packing in $G$ if for each $D$, there is a bound $M=M(D)$ on the number of distinct cosets $g_{i} H$ such that $d\left(g_{i} H, g_{j} H\right)<D$ for each $i, j$. (A more precise statement can be found in Section 2 below.) The connection between width and the bounded packing of a $\kappa$-quasiconvex subgroup $H$ of the $\delta$-hyperbolic group $G$, is that if $g_{i} H g_{i}^{-1} \cap g_{j} H g_{j}^{-1}$ is infinite, then $d\left(g_{i} H, g_{j} H\right)<K=K(\kappa, \delta)$.

The goal of this paper is to prove a bounded packing statement in the relatively hyperbolic context, and to deduce from this an appropriate finite width consequence.

Bounded packing is the fundamental notion operating behind proofs about the widths of subgroups and appears implicitly in Gitik-Mitra-Rips-Sageev [8]. It is a natural algebraic generalization of the Finite Plane Intersection Property (FPIP) for universal covers of surfaces in 3-manifolds (see, for instance, Rubinstein-Sageev [18]). It is also the key point used by Sageev [21] to prove the finite dimensionality of the cube complex arising from Sageev's construction applied to a codimension-1 quasiconvex subgroup of a word-hyperbolic group, as discussed in Section 3 below. In [11], the authors apply the bounded packing property to generalize Sageev's finite dimensional cubulation result to codimension-1 relatively quasiconvex subgroups of relatively hyperbolic groups. Our main theorem about bounded packing is the following result about relatively hyperbolic groups.

Theorem 1.1 Let $H$ be a relatively quasiconvex subgroup of a relatively hyperbolic group $G$. Suppose $H \cap g P g^{-1}$ has bounded packing in $\mathrm{gPg}^{-1}$ for each conjugate of each peripheral subgroup $P$. Then $H$ has bounded packing in $G$. 
This theorem generalizes a result of Gitik-Mitra-Rips-Sageev [8] on quasiconvex subgroups of a word hyperbolic group and also generalizes a result of RubinsteinSageev [18] on geometrically finite surfaces in 3-manifolds whose toral decomposition has only hyperbolic components.

We note that the condition about intersections with peripheral subgroups is necessary. Indeed, every group $G$ is hyperbolic relative to itself, and in that case every subgroup of $G$ is relatively quasiconvex.

We have collected together a number of basic properties of bounded packing in Section 2, and we state a variety of problems there for further thought. In general, we would like to know:

Question 1.2 Which countable groups have the property that all their (finitely generated) subgroups have bounded packing?

We also show in Section 3 that under mild hypotheses an action of a group $G$ on a CAT( 0 ) cube complex $C$ gives rise to natural subgroups of $G$ with bounded packing. These subgroups arise as stabilizers of hyperplanes in $C$.

\subsection{Pairwise close spaces}

Niblo-Reeves [13] proved that given $n, \kappa, D$ and $\delta$ there exists $M=M(n, \kappa, D, \delta)$ such that the following thinness condition holds: If $A_{1}, \ldots, A_{n}$ are $\kappa$-quasiconvex subspaces of a $\delta$-hyperbolic space $X$ such that $d\left(A_{i}, A_{j}\right)<D$ for all $i, j$, then there is a point $x \in X$ with $d\left(A_{i}, x\right)<M$ for all $i$. This statement is also implicit in Sageev's work in [21], and plays a fundamental role in both these papers in proving the cocompactness of the cube complex associated with a finite set of quasiconvex codimension-1 subgroups.

We generalize this statement in Proposition 7.7, which contains a slightly stronger conclusion than the following assertion that there is either a point or a peripheral coset nearby.

Proposition 1.3 Let $G$ be a relatively hyperbolic group. Choose positive constants $\kappa$ and $D$ and an integer $n \geq 1$. Let $\mathcal{A}$ be an arbitrary set of $\kappa$-relatively quasiconvex subspaces of $G$ such that for each $A, A^{\prime} \in \mathcal{A}$ the distance $d\left(A, A^{\prime}\right)$ is less than $D$. Then there is a constant $M=M(\kappa, D, n)$ so that at least one of the following holds.

(1) For every set $\left\{A_{1}, \ldots, A_{n}\right\}$ of $n$ distinct elements of $\mathcal{A}$, there is a point $x \in X$ such that $d\left(x, A_{i}\right)<M$ for each $i$.

(2) There is a peripheral coset $g P$ such that $d(g P, A)<M$ for all $A \in \mathcal{A}$.

Proposition 7.7 plays a significant role in our proof of Theorem 1.1. 


\subsection{Bounded height and width}

For relatively hyperbolic groups, the "small" subgroups are either "elliptic," meaning finite, or "parabolic," which means conjugate into a peripheral subgroup. In this context, we therefore redefine the height of $H$ in $G$ to be $(n-1)$ if $n$ is the smallest number with the property that for any $n$ distinct left cosets $g_{1} H, \ldots, g_{n} H$, the intersection $\bigcap_{1 \leq i \leq n} g_{i} H g_{i}^{-1}$ is elliptic or parabolic. Similarly, the width of $H$ in $G$ equals $(n-1)$ if $n$ is the smallest number with the property that for any $n$ distinct left cosets $g_{1} H, \ldots, g_{n} H$ the intersection $g_{i} H g_{i}^{-1} \cap g_{j} H g_{j}^{-1}$ is elliptic or parabolic for some $i, j$.

The following is our main theorem about finite height and width:

Theorem 1.4 Let $G$ be a relatively hyperbolic group, and let $H$ be a relatively quasiconvex subgroup. Then $H$ has finite height.

Suppose, furthermore that $H \cap g P g^{-1}$ has bounded packing in $g \mathrm{Pg}^{-1}$ for each conjugate of each peripheral subgroup $P$. Then $H$ has finite width.

We hope that further work in this area will resolve the following:

Problem 1.5 Let $G$ be relatively hyperbolic. Does every relatively quasiconvex subgroup of $G$ have finite width?

If $H$ is a subgroup of $G$, the commensurator of $H$ in $G$ is the subgroup of all $g \in G$ such that $H \cap \mathrm{gHg}^{-1}$ has finite index in both $H$ and $\mathrm{gHg}^{-1}$. We prove the following result on commensurators of relatively quasiconvex subgroups using ideas related to Theorem 1.4.

Theorem 1.6 Let $H$ be a relatively quasiconvex subgroup of a relatively hyperbolic group $G$. Suppose $H$ is infinite and nonparabolic. Then $H$ has finite index in its commensurator.

We use similar techniques to show that a separable (relatively) quasiconvex subgroup $H$ of a (relatively) hyperbolic group $G$ is (relatively) malnormal in a finite index subgroup $K$ of $G$ (see Theorem 9.3). This result is new even in the hyperbolic case.

\subsection{Overview}

In Section 2 we define the notion of bounded packing and prove a number of basic results about this concept. We also give a number of examples and collect several problems. 
In Section 3 we discuss the relation between bounded packing and actions on CAT(0) cube complexes. By a result of Sageev, a codimension-1 subgroup $H<G$ with bounded packing gives rise to an action of $G$ on a finite dimensional CAT(0) cube complex. In this section, we give a partial converse to Sageev's result.

In Section 4 we prove the bounded width theorem of Gitik-Mitra-Rips-Sageev [8] in the word hyperbolic setting. The reader is advised to understand this proof first, as our relatively hyperbolic generalization will follow it closely, albeit with certain aspects substantially more complicated.

In Section 5 we recount essential facts about the geometry of the Cayley graph of a relatively hyperbolic group with a finite generating set that were established by Druț-Sapir in [5]. We use these results to prove two auxiliary results about triangles and quadrilaterals with the property that each side lies near a peripheral subspace.

In Section 6 we discuss the geometry of relative Cayley graphs, concentrating on the interplay between the usual (proper) metric and the relative metric as applied to thinness conditions.

In Section 7 we analyze collections of relatively quasiconvex subspaces in a relatively hyperbolic group. In particular, we prove Proposition 7.7, an important technical result underlying this work that was described above.

In Section 8 we prove Theorem 1.4, our main result on bounded packing and bounded width for a relatively quasiconvex subgroup of a relatively hyperbolic group. We also prove Theorem 1.6 on the commensurator of a relatively quasiconvex subgroup.

Section 9 contains a brief proof of the relative malnormality result Theorem 9.3 mentioned above, using results from the previous section.

Acknowledgements The first author's contribution to this material is based upon work supported by the National Science Foundation under Grant Nos. DMS-0505659, DMS-0754254 and DMS-0808809. The second author is supported by grants from NSERC.

\section{Bounded packing: First properties and examples}

Definition 2.1 (Bounded packing) Let $G$ be a discrete group with a left invariant metric $d$. Suppose also that $d$ is proper in the sense that every metric ball is finite. A subgroup $H$ has bounded packing in $G$ (with respect to $d$ ) if, for each constant $D$, there is a number $N=N(G, H, D)$ so that for any collection of $N$ distinct cosets $g H$ in $G$, at least two are separated by a distance of at least $D$. 
A discrete group admitting a proper metric is necessarily countable. On the other hand, if $G$ is finitely generated then the word metric for any finite generating set is a proper, equivariant metric. Restricting the word metric on $G$ to a subgroup $H$ gives a proper, left invariant metric on $H$. Since every countable group is a subgroup of a finitely generated group, it follows that a discrete group $G$ admits a proper, left invariant metric if and only if $G$ is countable.

Lemma 2.2 If $G$ is countable, then bounded packing of a subgroup $H$ in $G$ is independent of the choice of proper, left invariant metric on $G$. In other words, if $d_{1}, d_{2}$ are proper, left invariant metrics on $G$, then $H$ has bounded packing in $G$ with respect to $d_{1}$ if and only if it has bounded packing with respect to $d_{2}$.

Proof Define $\rho: \mathbb{N} \rightarrow \mathbb{N}$ so that $\rho(n)$ is the supremum of $d_{1}(1, g)$ over all $g$ with $d_{2}(1, g)<n$. Note that there are only finitely many such $g$ since $d_{2}$ is proper, so $\rho(n)$ is finite for each $n$. By the left invariance of $d_{1}$ and $d_{2}$, it follows that

$$
d_{1}\left(g_{1}, g_{2}\right) \leq \rho\left(d_{2}\left(g_{1}, g_{2}\right)\right)
$$

for all $g_{1}, g_{2} \in G$.

Now if $\mathcal{H}$ is a collection of left cosets of $H$ with pairwise $d_{2}$-distances at most $D$, their pairwise $d_{1}$-distances are at most $\rho(D)$. Thus if $H$ has bounded packing with respect to $d_{1}$, it also has bounded packing with respect to $d_{2}$.

The following result follows easily from the definition of bounded packing.

Lemma 2.3 Any finite index subgroup $K$ of a countable group $G$ has bounded packing in $G$.

Proof The definition of bounded packing is vacuously satisfied once $N$ is larger than $[G: H]$, since there are no collections of $N$ distinct cosets of $H$ in $G$.

Lemma 2.4 Suppose that $H \leq K \leq G$ and $G$ is countable.

(1) If $H$ has bounded packing in $G$ then $H$ has bounded packing in $K$.

(2) If $H$ has bounded packing in $K$, and $K$ has bounded packing in $G$ then $H$ has bounded packing in $G$.

Proof We may assume the metric on $K$ is a restriction of the metric on $G$, so that (1) is immediate. 
Let $\mathcal{H}$ is a collection of left cosets of $H$ in $G$ with pairwise distances at most $D$. The bounded packing of $H$ in $K$ bounds the number of elements of $\mathcal{H}$ that lie in each left coset $g K$. Furthermore, the bounded packing of $K$ in $G$ bounds the number of cosets $g K$ that contain elements of $\mathcal{H}$. Thus the size of $\mathcal{H}$ is bounded in terms of $D$, establishing (2).

\section{Proposition 2.5 (Commensurability) Let $G$ be a countable group.}

(1) Suppose $H \leq K \leq G$ and $[K: H]<\infty$. Then $H$ has bounded packing in $G$ if and only if $K$ has bounded packing in $G$.

(2) Suppose $H \leq K \leq G$ and $[G: K]<\infty$. Then $H$ has bounded packing in $K$ if and only if $H$ has bounded packing in $G$.

(3) Suppose $H, K \leq G$ and $[G: K]<\infty$. Then $H \cap K$ has bounded packing in $K$ if and only if $H$ has bounded packing in $G$.

Proof Let $d$ be a proper, left invariant metric on $G$. To see (1) suppose $[K: H]<\infty$. Then there is a constant $C$ such that in the metric $d$ each left coset $g K$ is at most a Hausdorff distance $C$ from a left coset $g H$ (the choice of $g$ is irrelevant). Suppose $H$ has bounded packing in $G$. Let $\mathcal{K}$ be a collection of left cosets of $K$ with pairwise distances at most $D$. Replacing each coset $g K$ with a corresponding coset $g H$ gives a collection $\mathcal{H}$ of left cosets of $H$ with the same cardinality as $\mathcal{K}$ and with pairwise distances at most $D+2 C$. The bounded packing of $H$ in $G$ bounds the size of $\mathcal{H}$, and hence also the size of $\mathcal{K}$, as a function of $D$. Thus $K$ has bounded packing in $G$. Conversely suppose $K$ has bounded packing in $G$. By Lemma 2.3, we know that $H$ has bounded packing in $K$. Therefore $H$ has bounded packing in $G$ by Lemma 2.4(2), completing the proof of (1).

Assertion (2) follows immediately from Lemmas 2.3 and 2.4.

Suppose now that $H, K \leq G$ and $[G: K] \leq \infty$. Then $H \cap K$ has finite index in $H$. Observe that (3) is an immediate consequence of (1) and (2), since $H \cap K$ has bounded packing in $K$ if and only if $H \cap K$ has bounded packing in $G$ if and only if $H$ has bounded packing in $G$.

Corollary 2.6 If $G$ is countable, any finite subgroup $K \leq G$ has bounded packing in $G$.

Proof Fix a proper, left invariant metric $d$ on $G$. By Proposition 2.5(1) it suffices to show that the trivial subgroup has bounded packing in $G$. A left coset of the trivial subgroup is just a single element of $G$. If $\mathcal{H}$ is any collection of pairwise $D$-close 
left cosets of the trivial subgroup, (in other words, elements of $G$ ) then $\mathcal{H}$ is a subset of the ball of radius $D$ centered at any of its elements. The number of elements in such a ball is finite since $d$ is proper, and this number depends only on $D$ since $d$ is left-invariant.

Proposition 2.7 Suppose $H$ and $K$ have bounded packing in a countable group $G$. Then $H \cap K$ has bounded packing in $G$.

Proof Fix a proper, left invariant metric $d$ on $G$. By Lemmas 2.4(2) and 2.2 it suffices to show that $L:=H \cap K$ has bounded packing in $H$ with respect to $d$. Let $\mathcal{L}$ be a set of left cosets of $L$ in $H$ whose pairwise $d$-distances are at most $D$. If $h L$ and $h^{\prime} L$ are distinct cosets of $L$ in $H$ then $h L=H \cap h K$ and $h^{\prime} L=H \cap h^{\prime} K$ for distinct cosets $h K$ and $h^{\prime} K$ in $G$. Thus $\mathcal{L}$ is in one-to-one correspondence with a set $\hat{\mathcal{L}}$ of left cosets of $K$ in $G$ whose pairwise distances are at most $D$. But the bounded packing of $K$ in $G$ bounds the size of $\widehat{\mathcal{L}}$ as a function of $D$.

Lemma 2.8 Let $1 \rightarrow N \rightarrow G \rightarrow Q \rightarrow 1$ be a short exact sequence of countable groups. Let $H$ be a subgroup of $G$ which projects to the subgroup $\bar{H}$ of $Q$. Then $\bar{H}$ has bounded packing in $Q$ if and only if $H N$ has bounded packing in $G$.

Proof For each $g \in G$, let $\bar{g}$ denote the image of $g$ in $Q$. Fix a proper, left invariant metric $d$ on $G$. Define $\bar{d}: Q \rightarrow \mathbb{R}$ so that $\bar{d}\left(q_{1}, q_{2}\right)$ is the infimum of $d\left(g_{1}, g_{2}\right)$ over the set of all $g_{1}, g_{2}$ with $q_{i}=\bar{g}_{i}$. A straightforward argument shows that $\bar{d}$ is a proper, left invariant metric on $Q$.

The projection $G \rightarrow Q$ induces a one-to-one correspondence between left cosets of $H N$ in $G$ and left cosets of $\bar{H}$ in $Q$. It is clear that $d\left(g_{1}, g_{2}\right) \geq \bar{d}\left(\overline{g_{1}}, \overline{g_{2}}\right)$ for all $g_{1}, g_{2} \in G$. Therefore

$$
d(x H N, y H N) \geq \bar{d}(\bar{x} \bar{H}, \bar{y} \bar{H})
$$

for all $x, y \in G$.

Conversely, any element $x \in Q$ with $\bar{d}(x, \overline{1})=a$ lifts to an element $y \in G$ with $d(y, 1)=a$. If $\bar{d}\left(\overline{g_{1}} \bar{H}, \overline{g_{2}} \bar{H}\right)=n$ then there is an element $x \in Q$ with $\bar{d}(x, \overline{1})=a$ such that $\overline{g_{1}} \bar{H} \bar{w}$ intersects $\overline{g_{2}} \bar{H}$. If $y$ is a lift of $x$ with $d(y, 1)=a$, then $g_{1} H y N=$ $g_{1} H N y$ intersects $g_{2} H N$, so $d\left(g_{1} H N, g_{2} H N\right) \leq a$.

Therefore the distance between left cosets of $H N$ in $G$ is equal to the distance between corresponding left cosets of $\bar{H}$ in $Q$. Evidently $H N$ has bounded packing in $G$ if and only if $\bar{H}$ has bounded packing in $Q$. 
Corollary 2.9 Each normal subgroup $N$ of a countable group $G$ has bounded packing.

Proof Apply Lemma 2.8 with $H$ equal to the trivial subgroup.

Remark 2.10 In fact, a normal subgroup $N$ has the stronger property that for each $D$ there exists $M=M(D)$ such that at most $M$ left cosets $g N$ satisfy $d(N, g N)<D$.

Corollary 2.11 Every subgroup of a countable abelian group has bounded packing. $\square$

Theorem 2.12 Let $N$ be a countable, virtually nilpotent group. Then each subgroup of $N$ has bounded packing in $N$.

We note that this can be also be proven using either Lemma 2.17 or Corollary 2.15.

Proof By Proposition 2.5(3) it suffices to prove the theorem when $N$ is a countable nilpotent group. The theorem is obvious if $N$ is the trivial group. We proceed by induction on the length of the lower central series. Let $H$ be a subgroup of $N$. Let $Z$ denote the center of $N$. Let $\bar{N}:=N / Z$ and let $\bar{H}$ be the image of $H$ in $\bar{N}$. By induction, $\bar{H}$ has bounded packing in $\bar{N}$. Therefore by Lemma $2.8, H Z$ has bounded packing in $N$. Because $Z$ is central, $H$ is normal in $H Z$ and so $H$ has bounded packing in $H Z$ by Corollary 2.9. Finally, by Lemma 2.4(2), $H$ has bounded packing in $G$ since, $H$ has bounded packing in $H Z$ and $H Z$ has bounded packing in $G$.

Question 2.13 Let $S$ be a solvable group. Does every subgroup of $S$ have bounded packing in $S$ ?

It seems too much to expect an affirmative answer to Question 2.13, however we do expect the following to hold:

Conjecture 2.14 Let $P$ be virtually polycyclic. Then each subgroup of $P$ has bounded packing in $P$.

The following is an immediate consequence of Corollary 2.9 together with Lemma 2.4(2).

Corollary 2.15 Let $H$ be a subgroup of a countable group $G$. If $H$ is subnormal then $H$ has bounded packing in $G$. 
Example 2.16 For $n \neq 0$, let $\mathrm{BS}(1, n)$ be the group presented by $\left\langle a, t \mid t a t^{-1}=a^{n}\right\rangle$. It is well-known that for $n \neq \pm 1$, the subgroup $\langle a\rangle$ is not quasi-isometrically embedded; in fact, it is exponentially distorted. Nevertheless $\langle a\rangle$ has bounded packing in $\operatorname{BS}(1, n)$. Indeed, there is a short exact sequence $1 \rightarrow A \rightarrow \mathrm{BS}(1, n) \rightarrow \mathbb{Z} \rightarrow 1$ with $\langle a\rangle \leq A$ such that $A$ is isomorphic to $\mathbb{Z}[1 / n]$. Since $A$ is abelian, $\langle a\rangle$ is subnormal in $\operatorname{BS}(1, n)$ and hence has bounded packing by Corollary 2.15.

It is interesting to note that $A$ is not finitely generated. (Indeed, $A$ is locally cyclic but not itself cyclic.) Thus consideration of bounded packing in non-finitely generated groups can give us useful information about finitely generated subgroups of a finitely generated group.

As the following Lemma shows, the group $F_{2} \times \mathbb{Z}$ has bounded packing with respect to each of its finitely generated subgroups.

Lemma 2.17 Let $1 \rightarrow C \rightarrow G \rightarrow Q \rightarrow 1$ be a short exact sequence with $C$ central in the countable group $G$. If every (finitely generated) subgroup of $Q$ has bounded packing then every (finitely generated) subgroup of $G$ has bounded packing.

Proof Let $H$ be a [finitely generated] subgroup of $G$. By hypothesis, its image $\bar{H}$ has bounded packing in $Q$. Now $H$ has bounded packing in $C H$ by Corollary 2.9, and $C H$ has bounded packing in $G$ by Lemma 2.8. Therefore $H$ has bounded packing in $G$ by Lemma 2.4 .

Example 2.18 Let $M$ be a Seifert-fibered 3-manifold. Then there is a short exact sequence $1 \rightarrow \mathbb{Z} \rightarrow \pi_{1} M \rightarrow \pi_{1} S \rightarrow 1$, where $S$ is a 2 -dimensional orbihedron. Since every finitely generated subgroup of $\pi_{1} S$ has bounded packing (by local quasiconvexity and Theorem 4.8 below) it follows from Lemma 2.17 that every finitely generated subgroup of $\pi_{1} M$ has bounded packing.

Let us now examine subgroups that do not have bounded packing. At present surprisingly few such examples are known. In fact, the authors know of only one basic example, due to Rubinstein-Wang. The group is the fundamental group of a 3-dimensional graph manifold; that is, a manifold formed by gluing Seifert fibered manifolds together along tori. In light of the preceding example, we see that the bounded packing property is not preserved by very simple graphs of groups.

Example 2.19 Rubinstein-Wang [19] constructed a finitely generated surface subgroup of the fundamental group of a graph manifold that doesn't have bounded packing. Their example is an immersed $\pi_{1}$-injective surface $S$ in a 3 -dimensional graph 
manifold $M$. Let $\widetilde{S} \rightarrow \widetilde{M}$ denote a lift of $S \rightarrow M$ to a map between the universal covers. The various lifts correspond to translates $g \widetilde{S}$ where $g$ varies over the various cosets $g \pi_{1} S$. Remarkably, any two such lifts $g_{i} \tilde{S}$ and $g_{j} \tilde{S}$ intersect each other. Consequently any two cosets $g_{i} \pi_{1} S$ and $g_{j} \pi_{1} S$ are a uniformly bounded distance from each other in $\pi_{1} M$.

It seems reasonable to expect subgroups without bounded packing to appear in the fundamental groups of other graph manifolds. The following problem is a reasonable test case for this phenomenon.

Problem 2.20 Determine exactly which subgroups of the following group $G$ have bounded packing:

$$
G:=\langle a, b, c, d \mid[a, b],[b, c],[c, d]\rangle
$$

(The group $G$ is the fundamental group of a graph manifold.)

Since there are subgroups of countable groups without bounded packing, it follows from Lemma 2.8 that (non-finitely generated) subgroups of the free group $F_{2}$ do not always have bounded packing. Notice that, since finitely generated subgroups of $F_{2}$ are quasiconvex, Theorem 4.8 below implies that finitely generated subgroups of $F_{2}$ always have bounded packing.

Rips gave the following construction in [16]:

Proposition 2.21 Let $Q$ be a finitely presented group. Then there exists a short exact sequence $1 \rightarrow N \rightarrow G \rightarrow Q \rightarrow 1$ such that $N$ is finitely generated, and $G$ is a finitely presented $C^{\prime}\left(\frac{1}{6}\right)$ group.

Example 2.22 Using Rips's construction, we will construct a word hyperbolic group $G$ and a finitely generated subgroup $K$ such that $K$ does not have bounded packing in $G$. Let $Q$ be a finitely presented group with a subgroup $H$ that fails to have bounded packing in $Q$. Let $1 \rightarrow N \rightarrow G \rightarrow Q \rightarrow 1$ be the short exact sequence from Proposition 2.21. Let $K$ be the preimage of $H$ in $G$, and note that $K$ is finitely generated since both $N$ and $H$ are. By Lemma 2.8, $K$ does not have bounded packing in $G$. Observe that $G$ is word hyperbolic, since it is finitely presented and $C^{\prime}\left(\frac{1}{6}\right)$.

Remark 2.23 We can choose $H$ to be a codimension-1 subgroup without bounded packing to make the group $G$ in Example 2.22 have an exotic action on an infinite dimensional cube complex.

Problem 2.24 Give an example of a cyclic subgroup $Z$ of a finitely generated group $G$ such that $Z$ does not have bounded packing in $G$. 
Question 2.25 Are there finitely generated subgroups of $F_{2} \times F_{2}$ without bounded packing?

Question 2.26 Let $1 \rightarrow N \rightarrow G \rightarrow A \rightarrow 1$ be an extension of finitely generated groups. Suppose every subgroup of $N$ has bounded packing in $N$, and $A$ is abelian. Does every subgroup of $G$ have bounded packing in $G$ ?

Problem 2.27 Let $H$ be a retract of the finitely generated group $G$. Does $H$ have bounded packing in $G$ ?

\section{Bounded packing and actions on $\mathrm{CAT}(0)$ cube complexes}

Suppose $G$ is a group with a finite generating set $\mathcal{S}$. A subgroup $H<G$ is codimension1 if there is a constant $L>0$ so that the $L$-neighborhood of $H$ in Cayley $(G, \mathcal{S})$ has at least two "deep" complementary components. A complementary component is deep if it contains elements whose distance from $H$ is arbitrarily large.

A theorem of Sageev [20], together with a result proved independently by Gerasimov [7] and Niblo-Roller [14], shows that a group $G$ with a codimension-1 subgroup $H$ has an action on a CAT(0) cube complex $C$ without a global fixed point. All hyperplanes in $C$ lie in a single orbit under the action of $G$, and $H$ is the stabilizer of some hyperplane $\Lambda$. The dimension of $C$ is the maximal size of a set of pairwise transverse hyperplanes. The relation to bounded packing is the following basic fact, observed by Sageev [21]: If $H$ is finitely generated and hyperplanes $g_{0} \Lambda$ and $g_{1} \Lambda$ in $C$ are transverse, then $d_{\mathcal{S}}\left(g_{0} H, g_{1} H\right)<M$ for some universal constant $M$.

Corollary 3.1 (Sageev) Suppose $H$ is a finitely generated codimension-1 subgroup of a finitely generated group $G$. If $H$ has bounded packing in $G$, then the corresponding CAT(0) cube complex $C$ is finite dimensional.

The goal of this section is to prove the following converse, of sorts, to the preceding result.

Theorem 3.2 Suppose the countable discrete group $G$ acts on a CAT(0) cube complex $C$, and $H \leq G$ is the stabilizer of a hyperplane $\Lambda$.

(1) If $C$ is locally finite, then $H$ has bounded packing in $G$.

(2) Let $V(\Lambda)$ be the set of vertices incident to the edges that cross $\Lambda$. If $G$ acts metrically properly on $C$, and $H$ acts on $V(\Lambda)$ with only finitely many orbits of vertices, then $H$ has bounded packing in $G$. 
In a sense this theorem is optimal, as shown by the following example.

Example 3.3 Recall that Example 2.19 gives a group $G$ and a subgroup $H$ such that $H$ does not have bounded packing in $G$. The group $H$ is the fundamental group of an immersed surface $S$ in a 3-dimensional graph manifold $M$ with $G=\pi_{1}(M)$. Since $H$ is a codimension-1 subgroup of $G$, it follows that $G$ has an action on a CAT(0) cube complex $C$ such that $H$ is the stabilizer of a hyperplane $\Lambda$. Condition (1) fails resolutely since $C$ is an infinite dimensional cube, as any two translates of the surface $S$ cross. The authors believe failure of (2) can be traced to a failure of the cocompactness of the action of $H$ on $V(\Lambda)$. Indeed even if the action of $G$ on $C$ is not proper, the authors believe this can be remedied with the addition of further codimension-1 subgroups leading to a metrically proper action on a new cube complex $C^{\prime}$. The action of $H$ on the new hyperplane $\Lambda^{\prime}$ would necessarily fail to be cocompact by Theorem 3.2, as $H$ does not have bounded packing in $G$.

Theorem 3.2 follows from several results about convex subsets of a $\operatorname{CAT}(0)$ cube complex $C$. Let $C^{1}$ denote the 1 -skeleton of a CAT( 0$)$ cube complex with its induced path metric $d$. It is well-known that the metric $d$ on the vertices coincides with the "wall metric." That is to say, $d(v, w)$ is equal to the number of hyperplanes separating $v$ from $w$. The interval $[v, w]$ is the set of all vertices $u$ that lie on some $d$-geodesic from $v$ to $w$. A subset $S \subseteq C^{0}$ is $d$-convex if every interval connecting two elements of $S$ is contained in $S$.

We also find it useful to consider the graph $C^{\Delta}$ obtained from $C^{1}$ by including an edge between two vertices if they lie in a common cube. Let $d^{\Delta}$ be the path metric on $C^{\Delta}$.

Lemma 3.4 Let $C$ be a CAT(0) cube complex, and let $[r, s]$ and $[t, u]$ be intervals such that $t$ and $u$ lie in the 1 -neighborhood of $[r, s]$ in $C^{\Delta}$. Then each vertex of $[t, u]$ lies in the 1-neighborhood of $[r, s]$ in $C^{\Delta}$ as well.

Proof It is enough to show that any geodesic $c$ from $t$ to $u$ lies in the 1-neighborhood of $[r, s]$ in $C^{\Delta}$ if its endpoints lie in the 1-neighborhood of $[r, s]$ in $C^{\Delta}$. If $d(t, u) \leq 1$, the result is trivial, so we induct on $\ell:=d(t, u)$ for $\ell \geq 2$.

By hypothesis, there is a cube $A$ containing $t$ and intersecting $[r, s]$. Let us choose $A$ minimal with this property. Then the hyperplanes $\Lambda_{1}, \ldots, \Lambda_{n}$ separating $t$ from $[r, s]$ are the same as the hyperplanes transverse to $A$. (In particular, $A$ is an $n$-cube.)

Let $v$ be the vertex of $c$ adjacent to $t$, and let $\Pi$ be the hyperplane separating $v$ from $t$. If $\Pi=\Lambda_{i}$ for some $i$, then $v$ is a vertex of the cube $A$, and we are done by induction. 
If $\Pi$ is transverse with $\Lambda_{i}$ for all $i$, then the collection $\left\{\Pi, \Lambda_{1}, \ldots, \Lambda_{n}\right\}$ is pairwise transverse. Since $\Pi$ and the $\Lambda_{i}$ are each transverse to edges incident to $t$, there is an $(n+1)$-cube $A^{\prime}$ containing $A$ as a subcube, such that $\Pi$ and each $\Lambda_{i}$ are transverse to $A^{\prime}$. Since $A$ intersects $[r, s]$ and $A^{\prime}$ contains $v$, the result follows from the inductive hypothesis.

The only remaining possibility is that $\Pi$ is nested with some $\Lambda_{i} \neq \Pi$. Then $\Lambda_{i}$ separates $[r, s]$ from $\{t, v\}$ and $\Pi$ separates $[r, s] \cup\{t\}$ from $\{v\}$. In this case, any path in $C^{1}$ from $v$ to $[r, s]$ must cross $\Pi$ first and then $\Lambda_{i}$. Since $c$ is a geodesic in the wall metric, it crosses $\Pi$ only once, so $\Pi$ lies in the pairwise transverse family $\left\{\Pi_{1}, \ldots, \Pi_{m}\right\}$ of hyperplanes separating $u$ from $[r, s]$, corresponding to a minimal cube $B$ that contains $u$ and intersects $[r, s]$. But then $\Lambda_{i}$ is also among the $\Pi_{j}$, contradicting our assumption that $\Lambda_{i}$ and $\Pi$ are nested.

Corollary 3.5 Let $C$ be a CAT(0) cube complex, and let $K$ be a $d$-convex subset of $C^{0}$. For each $n \geq 0$, the $n$-neighborhood of $K$ in $C^{\Delta}$ is also $d$-convex.

Proof It is clearly sufficient to prove the result when $n=1$, since the general result follows by induction on $n$. But the case $n=1$ is an immediate consequence of Lemma 3.4 .

A collection of subsets $\mathcal{F}$ of a space has the Helly property if, whenever $\mathcal{F}_{0} \subseteq \mathcal{F}$ is a finite collection of pairwise intersecting subsets, the total intersection $\bigcap_{F \in \mathcal{F}_{0}} F$ is nonempty.

Corollary 3.6 If $C$ is any CAT( 0$)$ cube complex, then the family of $d$-convex subsets of $C^{0}$ has the Helly property.

Proof It has been shown by Gerasimov [7], Roller [17] and Chepoi [4], independently, that the 1 -skeleton of a $\operatorname{CAT}(0)$ cube complex is a median graph, meaning that for any three vertices $x, y$ and $z$, the intervals $[x, y],[y, z]$ and $[x, z]$ have triple intersection consisting of a single vertex. In a median graph, it is clear from the definition that any collection of three pairwise intersecting convex sets has a nonempty triple intersection. The Helly property now follows by an elementary induction argument.

The previous result generalizes a result of Bandelt and van de Vel [2] in the setting of median graphs stating that the collection of all balls in $C^{\Delta}$ has the Helly property. Note that $d^{\Delta}$-balls are $d$-convex by Corollary 3.5. Graphs in which the family of balls has the Helly property are known as Helly graphs.

We also record the following well-known fact about hyperplanes, which we derive from results above. 
Proposition 3.7 Let $\Lambda$ be a hyperplane in a CAT( 0$)$ cube complex, and let $V(\Lambda)$ be the set of vertices of the edges crossing $\Lambda$. Then $V(\Lambda)$ is $d$-convex.

Proof Each halfspace of $\Lambda$ is clearly $d$-convex. But $V(\Lambda)$ is the set of points within a $d^{\Delta}$-distance 1 of both halfspaces of $\Lambda$. Since $d^{\Delta}$-neighborhoods of $d$-convex sets are convex and intersections of $d$-convex sets are $d$-convex, the result is obvious. $\square$

We are now ready to prove Theorem 3.2.

Proof of Theorem 3.2 Suppose $H$ stabilizes the hyperplane $\Lambda$ in $C$. Let $V(\Lambda)$ be the convex set of vertices of the edges that cross $\Lambda$. Fix a proper, left invariant metric $d_{G}$ on $G$ and a constant $D>0$. Suppose $\mathcal{H}$ is a finite collection of cosets of $H$ whose pairwise distances in $G$ are at most $D$.

Choose a basepoint $x \in V(\Lambda)$, and let

$$
D^{\prime}:=\sup d^{\Delta}(x, g(x))
$$

where the supremum ranges over the finite set of group elements whose $d_{G}$-distance from 1 is at most $D$. If cosets $g H$ and $g^{\prime} H$ are at a distance at most $D$ in $G$, then the sets $g H(x) \subset V(g \Lambda)$ and $g^{\prime} H(x) \subset V\left(g^{\prime} \Lambda\right)$ are at a $d^{\Delta}$-distance at most $D^{\prime}$. Therefore the translates $V(g \Lambda)$ and $V\left(g^{\prime} \Lambda\right)$ are at a $d^{\Delta}$-distance at most $D^{\prime}$ as well. It follows immediately that the $D^{\prime}$-neighborhoods of the translates $V(g \Lambda)$ are pairwise intersecting $d$-convex sets in $C$. Hence there is a point $p$ within a $d^{\Delta}$-distance $D^{\prime}$ of every $V(g \Lambda)$.

If $C$ is uniformly locally finite, then there is a uniform bound on the number of hyperplanes intersecting any $d^{\Delta}$-ball of radius $D^{\prime}$ in $C$. Hence the cardinality of $\mathcal{H}$ is bounded as well.

Now suppose $G$ acts metrically properly on $C$, and $H$ acts on $V(\Lambda)$ with a finite quotient. Then $V(\Lambda)$ lies in the $R$-neighborhood of the orbit $H x$ in $C^{\Delta}$ for some $R>0$. Thus the $d^{\Delta}$-ball centered at $p$ with radius $D^{\prime}+R$ intersects $g H(x)$ for each coset $g H \in \mathcal{H}$. Since $G$ acts metrically properly on $C$, there is a finite upper bound on the size of $\mathcal{H}$, as desired.

\section{Bounded packing in hyperbolic groups}

In this section, we give a self-contained proof in the word hyperbolic setting that quasiconvex subgroups have bounded packing. 
Definition 4.1 Let $H \leq G$ be a subgroup. The height of $H$ in $G$, denoted height ${ }_{G}(H)$, is the maximal number of distinct cosets $g_{i} H$ so that the intersection $\bigcap_{i} g_{i} H_{i}^{-1}$ is infinite. The width of $H$ in $G$, denoted width $G(H)$, is the maximal number of distinct cosets $g_{i} H$ so that for all $i, j$ the intersection $g_{i} H g_{i}^{-1} \cap g_{j} H g_{j}^{-1}$ is infinite. In case there is no maximum then we say that the height/width is infinite.

Finally, note that the height and width of a finite subgroup are both equal to zero.

Lemma 4.2 Suppose $H \leq G$ has height $0<n<\infty$ in $G$. Choose $g \in G$ so that $g H \neq H$, and let $K:=H \cap g H^{-1}$. Then height $_{H}(K)<n$.

Proof Choose $h_{1}, \ldots, h_{n} \in H$ so that the cosets $h_{i} K$ are distinct. We will show that the intersection $\bigcap h_{i} K h_{i}^{-1}$ is finite. Note that

$$
\bigcap h_{i} K h_{i}^{-1}=\bigcap h_{i}\left(H \cap g H g^{-1}\right) h_{i}^{-1}=H \cap\left(\bigcap h_{i} g H g^{-1} h_{i}^{-1}\right)
$$

is an intersection of $n+1$ conjugates of $H$ in $G$. Since height ${ }_{G}(H)=n$, it suffices to show that the elements $1, h_{1} g, \ldots, h_{n} g$ represent distinct left cosets of $H$ in $G$.

First note that $H \neq h_{i} g H$ since $H \neq g H$. Now suppose two cosets $h_{i} g H$ and $h_{j} g H$ are equal. Then we have $g H=h_{i}^{-1} h_{j} g H$, which implies that $h_{i}^{-1} h_{j} \in g H^{-1}$. But $h_{i}^{-1} h_{j} \in H$ as well, so $h_{i}^{-1} h_{j} \in H \cap g H g^{-1}=K$. Thus $h_{i} K=h_{j} K$, and we must have $i=j$.

Lemma 4.3 Let $Y$ be a $\kappa$-quasiconvex subspace of a $\delta$-hyperbolic space $X$. Then there exists $\kappa^{\prime}=\kappa^{\prime}(\kappa, \delta)$ so that any geodesic line $A$ in a finite neighborhood of $Y$ lies in a $\kappa^{\prime}$-neighborhood of $Y$.

Proof Let $c$ be an arbitrary line in a finite neighborhood of $Y$. Then the endpoints $c^{+}$and $c^{-}$of $c$ lie in the limit set of $Y$. Let $c^{\prime}$ be a line connecting $c^{+}$and $c^{-}$that is a limit of geodesic segments connecting points of $Y$. In a $\delta$-hyperbolic space, two geodesic lines with the same endpoints at infinity are at a Hausdorff distance at most $2 \delta$. But by quasiconvexity, $c^{\prime} \subseteq \mathcal{N}_{\kappa}(Y)$, so $c \subseteq \mathcal{N}_{\kappa+2 \delta}(Y)$.

Lemma 4.4 Let $H$ be a $\kappa$-quasiconvex subgroup of a $\delta$-hyperbolic group $G$. Then height $_{G}(H)$ is finite.

Proof Let $g_{1} H, \ldots g_{n} H$ be distinct cosets in $G$, and suppose $\bigcap g_{i} H g_{i}^{-1}$ contains an infinite order (hyperbolic) element $x$. Let $A$ be a geodesic axis for $x$. Then $A$ lies in a finite neighborhood of each coset $g_{i} H$, so by Lemma 4.3 the size of these neighborhoods is uniformly bounded by $\kappa^{\prime}$. In particular, each coset $g_{i} H$ intersects the ball of radius $\kappa^{\prime}$ about some point $a \in A$. But there is a uniform bound $N$ on the number of cosets of $H$ intersecting any metric ball of radius $\kappa^{\prime}$. 
Lemma 4.5 Let $G$ have a finite generating set $\mathcal{A}$, and suppose $x H$ and $y K$ are arbitrary left cosets of subgroups of $G$. For each constant $L$ there is a constant $L^{\prime}=L^{\prime}(G, \mathcal{A}, x H, y K)$ so that in $G$ with the word metric $d_{\mathcal{A}}$ we have:

$$
\mathcal{N}_{L}(x H) \cap \mathcal{N}_{L}(y K) \subseteq \mathcal{N}_{L^{\prime}}\left(x H x^{-1} \cap y K y^{-1}\right)
$$

Proof If there is no such $L^{\prime}$, then there is a sequence $\left(z_{i}\right)$ in $G$ so that $z_{i}$ is in the $L$-neighborhood of both $x H$ and $y K$, but $i<d\left(z_{i}, x H x^{-1} \cap y K y^{-1}\right)$ for each $i$. It follows that $z_{i}=x h_{i} p_{i}=y k_{i} q_{i}$ for some $h_{i} \in H, k_{i} \in K$ and $p_{i}, q_{i} \in G$ with $\left|p_{i}\right|_{\mathcal{A}}$ and $\left|q_{i}\right|_{\mathcal{A}}$ both less than $L$. Passing to a subsequence if necessary, we may assume that $p_{i}$ and $q_{i}$ are constants $p$ and $q$, so that for each $i$ we have $z_{i}=x h_{i} p=y k_{i} q$. Therefore

$$
z_{i} z_{1}^{-1}=x h_{i} h_{1}^{-1} x^{-1}=y k_{i} k_{1}^{-1} y^{-1} \in x H x^{-1} \cap y K y^{-1} .
$$

It follows that the distance between $z_{i}$ and $x H x^{-1} \cap y K y^{-1}$ is at most $\left|z_{1}\right|_{\mathcal{A}}$ for all $i$, contradicting our choice of $\left(x_{i}\right)$.

The following result follows immediately by induction from Lemma 4.5.

Corollary 4.6 Let $G$ be a finitely generated group with a fixed generating set $\mathcal{A}$. For each finite set of cosets $g_{1} H_{1}, \ldots g_{n} H_{n}$ and each $M>0$ there is a constant $M^{\prime}>0$ such that:

$$
\mathcal{N}_{M}\left(g_{1} H_{1}\right) \cap \cdots \cap \mathcal{N}_{M}\left(g_{n} H_{n}\right) \subseteq \mathcal{N}_{M^{\prime}}\left(g_{1} H_{1} g_{1}^{-1} \cap \cdots \cap g_{n} H_{n} g_{n}^{-1}\right)
$$

Lemma 4.7 Let $X$ be a $\delta$-hyperbolic space, and let $Y$ be a $\kappa$-quasiconvex subspace. For any $\xi>0$ there is an $\eta=\eta(\delta, \kappa, \xi)$ so that the $\xi$-neighborhood of $Y$ is $\eta-$ quasiconvex.

Proof Choose points $x, y \in \mathcal{N}_{\xi}(Y)$, and $z, w \in Y$ so that $d(x, z)$ and $d(y, w)$ are less than $\xi$. Since quadrilaterals in $X$ are $2 \delta$-thin, the geodesic $[x, y]$ lies in the $2 \delta$-neighborhood of $[x, z] \cup[z, w] \cup[w, y]$. But this piecewise geodesic lies in the $(\kappa+\eta)$-neighborhood of $Y$.

Theorem 4.8 (cf Gitik-Mitra-Rips-Sageev [8]) Let $H$ be a quasiconvex subgroup of a $\delta$-hyperbolic group $G$. Then $H$ has bounded packing in $G$.

Before giving the proof of Theorem 4.8, we record the following:

Corollary 4.9 (Gitik-Mitra-Rips-Sageev [8]) If $H$ is a quasiconvex subgroup of a $\delta$-hyperbolic group $G$, then width $_{G}(H)$ is finite.

Proof As in Lemma 4.4, apply Lemma 4.3 to see that any two conjugates with infinite intersection have cosets uniformly close together. 
Proof of Theorem 4.8 Fix finite generating sets $\mathcal{A}$ and $\mathcal{B}$ for $G$ and $H$ respectively. By Lemma 4.4, we know that height ${ }_{G}(H)$ is finite. We will prove the theorem by induction on height. The case where $H$ has height zero is easy, since in this case $H$ is finite and the result follows from Corollary 2.6.

Now assume by induction that the theorem holds for every hyperbolic group $G^{\prime}$ and quasiconvex subgroup $H^{\prime}$ with height $G_{G^{\prime}}\left(H^{\prime}\right)<$ height $_{G}(H)$. Let $\mathcal{H}$ be a set of left cosets $g H$ whose pairwise distances are at most $D$. Our goal is to show that $\mathcal{H}$ is finite and to bound the cardinality of $\mathcal{H}$ as a function of $D$. Translating $\mathcal{H}$ if necessary, we may assume that $H \in \mathcal{H}$. Observe that if $d(g H, H)<D$ then the coset $h g H$ is within a distance $D$ of the identity for some $h \in H$. Since the metric $d_{\mathcal{A}}$ is proper on $G$, the ball of radius $D$ centered at the identity is finite. It follows that the left cosets $g H$ intersecting $\mathcal{N}_{D}(H)$ lie in at most $f$ distinct $H$-orbits for some $f=f(D)<\infty$. Thus it suffices to bound the number of elements of $\mathcal{H}$ in the orbit $H(g H)$ for each fixed $g \notin H$.

If we let $K:=H \cap g \mathrm{Hg}^{-1}$, then Lemma 4.2 shows that

$$
\text { height }_{H}(K)<\text { height }_{G}(H) \text {. }
$$

Since $K$ is a quasiconvex subgroup of the hyperbolic group $H$, the inductive hypothesis applied to $K \leq H$ gives for each $D^{\prime}$ a number $M^{\prime}=M^{\prime}\left(D^{\prime}\right)<\infty$ so that any collection of $M^{\prime}$ distinct cosets $h K$ in $H$ contains a pair separated by a $\mathcal{B}$-distance at least $D^{\prime}$. Furthermore, the proof of Lemma 4.2 shows that there is a well-defined map $h g H \rightarrow h K$ taking left cosets of $H$ in the orbit of $g H$ to left cosets of $K$. A similar argument shows that this map is bijective.

In order to complete the proof, we will show that $D$-closeness of distinct cosets $h_{0} g H$ and $h_{1} g H$ in $\left(G, d_{\mathcal{A}}\right)$ implies $D^{\prime}$-closeness of the corresponding cosets $h_{0} K$ and $h_{1} K$ in $\left(H, d_{\mathcal{B}}\right)$ for some $D^{\prime}$ depending on $D$. Roughly speaking, this claim is proved by considering a $\delta$-thin triangle in $G$ whose sides are close to the three cosets $H$, $h_{0} g H$ and $h_{1} g H$, and applying Lemma 4.5. More precisely, suppose we have points $x, y$ and $z$ such that

and

$$
\begin{aligned}
& x \in \mathcal{N}_{D}(H) \cap \mathcal{N}_{D}\left(h_{0} g H\right), \\
& y \in \mathcal{N}_{D}(H) \cap \mathcal{N}_{D}\left(h_{1} g H\right) \\
& z \in \mathcal{N}_{D}\left(h_{0} g H\right) \cap \mathcal{N}_{D}\left(h_{1} g H\right) .
\end{aligned}
$$

By Lemma 4.7 we have

$$
[x, y] \subset \mathcal{N}_{D_{1}}(H), \quad[x, z] \subset \mathcal{N}_{D_{1}}\left(h_{0} g H\right) \quad \text { and } \quad[y, z] \subset \mathcal{N}_{D_{1}}\left(h_{1} g H\right)
$$


for some $D_{1}$ depending on $D$. By $\delta$-hyperbolicity of $\left(G, d_{\mathcal{A}}\right)$, there is a point $w \in[x, y]$ within a distance $\delta$ of both $[x, z]$ and $[y, z]$. Applying the isometry $h_{i}$ to the conclusion of Lemma 4.5, we see that

$$
\mathcal{N}_{D_{1}}(H) \cap \mathcal{N}_{D_{1}+\delta}\left(h_{i} g H\right) \subseteq \mathcal{N}_{D_{2}}\left(h_{i} K\right),
$$

where $D_{2}$ depends on $D_{1}$ but is independent of the choice of $h_{i} \in H$. In other words, $w$ lies within an $\mathcal{A}$-distance $D_{2}$ of both $h_{1} K$ and $h_{2} K$ in $G$. Since $H$ is an undistorted subgroup of $G$, it follows that the $\mathcal{B}$-distance between $h_{1} K$ and $h_{2} K$ in $H$ is similarly bounded in terms of $D$, as desired.

\section{Relatively hyperbolic groups and the word metric}

Various equivalent formulations of relatively hyperbolic groups have been introduced and studied by Gromov [9], Farb [6], Bowditch [3], Druțu-Sapir [5] and Osin [15].

In this section we discuss geometric features of the Cayley graph of a relatively hyperbolic group with respect to a finite generating set. In particular, we prove two results about triangles and quadrilaterals with the property that each side lies near a peripheral subspace.

We begin by recalling the definition of a relatively hyperbolic group.

Definition 5.1 (Relatively hyperbolic) Let $G$ be a finitely generated group and $\mathbb{P}$ a finite collection of subgroups of $G$. Suppose $G$ acts on a $\delta$-hyperbolic graph $K$ with finite edge stabilizers and finitely many orbits of edges. Suppose $K$ is fine in the sense that for each $n$ each edge of $K$ is contained in only finitely many circuits of length $n$. Suppose also that $\mathbb{P}$ is a set of representatives of the conjugacy classes of infinite vertex stabilizers. Then the pair $(G, \mathbb{P})$ is relatively hyperbolic. The subgroups $P \in \mathbb{P}$ are the peripheral subgroups of $(G, \mathbb{P})$, and their left cosets $g P$ are peripheral cosets.

Throughout this section all paths and distances are taken in the Cayley graph of a relatively hyperbolic group with respect to a fixed finite generating set.

We now collect several results due to Druț-Sapir on the geometry of the word metric for a relatively hyperbolic group. The first states that peripheral cosets are "isolated."

Theorem 5.2 (Druț-Sapir [5, Theorem 4.1]) Suppose $(G, \mathbb{P})$ is relatively hyperbolic. For each $\rho<\infty$ there is a constant $\kappa=\kappa(\rho)<\infty$ so that for any two peripheral cosets $g P \neq g^{\prime} P^{\prime}$ we have

$$
\operatorname{diam}\left(\mathcal{N}_{\rho}(g P) \cap \mathcal{N}_{\rho}\left(g^{\prime} P^{\prime}\right)\right)<\kappa .
$$


The next result quantifies the quasiconvexity of peripheral cosets with respect to quasigeodesics.

Theorem 5.3 (Druțu-Sapir [5, Lemma 4.15]) Suppose $(G, \mathbb{P})$ is relatively hyperbolic. Given positive constants $\epsilon$ and $\nu$, there is a constant $\tau=\tau(\epsilon, \nu)$ so that the following holds. Let $c$ be an $\epsilon$-quasigeodesic with endpoints in the $v$-neighborhood of a peripheral coset $g P$. Then $c \subseteq \mathcal{N}_{\tau}(g P)$.

The following proposition roughly states that a pair of quasigeodesics beginning in the same peripheral coset and ending in the same peripheral coset must leave the former coset and enter the latter coset close together.

Proposition 5.4 (Druțu-Sapir [5, Lemma 8.11]) Let $(G, \mathbb{P})$ be relatively hyperbolic. There is a constant $v_{0}$ such that for each $v>v_{0}$ there is a constant $D_{1}=D_{1}(v)$ such that the following holds. Let $g P$ and $g^{\prime} P^{\prime}$ be two distinct peripheral cosets and let $c_{1}$ and $c_{2}$ be two geodesics such that $c_{i}$ has endpoints $x_{i}$ and $y_{i}$. If $c_{i} \cap \overline{\mathcal{N}_{v}(g P)}=\left\{x_{i}\right\}$ and $c_{i} \cap \overline{\mathcal{N}_{v}\left(g^{\prime} P^{\prime}\right)}=\left\{y_{i}\right\}$ then $d\left(x_{1}, x_{2}\right)$ and $d\left(y_{1}, y_{2}\right)$ are both less than $D_{1}$.

Druț-Sapir also establish the following geometric description of quasigeodesic triangles.

Theorem 5.5 (Druțu-Sapir [5, Section 8.1.3]) Let $(G, \mathbb{P})$ be relatively hyperbolic. For each $\epsilon$ there are constants $\delta=\delta(\epsilon)$ and $\mu=\mu(\epsilon)$ such that the following holds. Let $\Delta$ be an $\epsilon$-quasigeodesic triangle with sides $c_{0}, c_{1}$ and $c_{2}$. Then either

(1) there is a point $w$ such that the ball $B(w, \delta)$ intersects all three sides of $\Delta$, or

(2) there is a peripheral coset $g P$ such that the neighborhood $\mathcal{N}_{\delta}(g P)$ intersects all three sides of $\Delta$.

In the second case, illustrated in Figure 1, let $c_{i}^{\prime}$ be the smallest subpath of $c_{i}$ containing $c_{i} \cap \mathcal{N}_{\delta}(g P)$. Then the terminal endpoint of $c_{i}^{\prime}$ and the initial endpoint of $c_{i+1}^{\prime}$ are mutually within a distance $\mu$ (indices modulo 3 ).

Roughly speaking, the following lemma deals with a triangle each of whose sides lies close to a peripheral coset. The conclusion is that either all three peripheral cosets are equal or one of the sides of the triangle is short.

Lemma 5.6 Let $(G, \mathbb{P})$ be relatively hyperbolic. For each $\eta>0$, there is a constant $\zeta>0$ such that the following holds. Let $g_{0} P_{0}, g_{1} P_{1}$ and $g_{2} P_{2}$ be peripheral cosets such that $g_{0} P_{0} \notin\left\{g_{1} P_{1}, g_{2} P_{2}\right\}$. Suppose for each $\{i, j, k\}=\{0,1,2\}$ there is a point

$$
y_{i} \in \mathcal{N}_{\eta}\left(g_{j} P_{j}\right) \cap \mathcal{N}_{\eta}\left(g_{k} P_{k}\right) .
$$

Then

$$
d\left(y_{1}, y_{2}\right)<\zeta .
$$




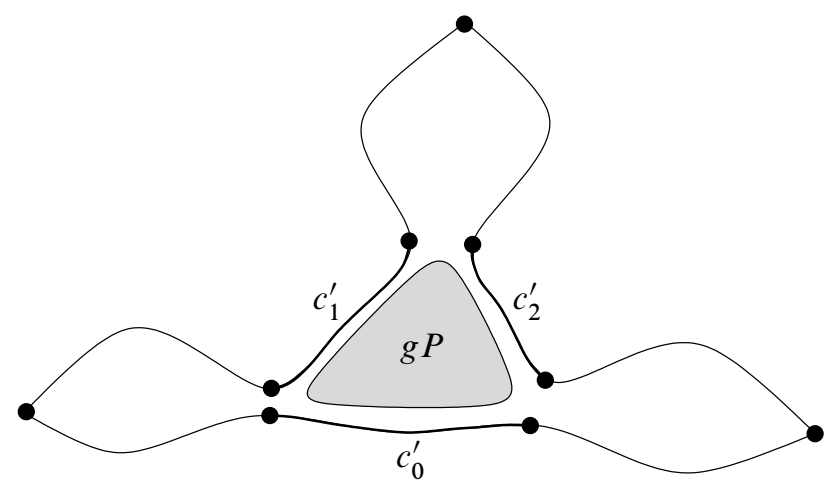

Figure 1: A quasigeodesic triangle in a relatively hyperbolic group with a peripheral coset at the center

Proof For each choice of $\{i, j, k\}=\{0,1,2\}$, pick a point $y_{i} \in \mathcal{N}_{\eta}\left(g_{j} P_{j}\right) \cap \mathcal{N}_{\eta}\left(g_{k} P_{k}\right)$. Consider a geodesic triangle $\Delta:=c_{0} \cup c_{1} \cup c_{2} \cup$ such that each $c_{i}$ has endpoints $y_{j}$ and $y_{k}$. By Theorem 5.3, there is a constant $\tau$ depending on $\eta$ such that $c_{i}$ lies in the $\tau$-neighborhood of $g_{i} P_{i}$.

Let $\delta=\delta(1)$ and $\mu=\mu(1)$ be the constants given by Theorem 5.5. Then there is either a point $z$ or a peripheral $\operatorname{coset} g P$ whose $\delta$-neighborhood intersects all three sides of $\Delta$.

We will see that we must be in the former case, for a possibly larger constant $\delta^{\prime}$. Suppose $\mathcal{N}_{\delta}(g P)$ intersects all three sides of $\Delta$. For each $i$, let $c_{i}^{\prime}$ denote the smallest subpath of $c_{i}$ containing $c_{i} \cap \overline{\mathcal{N}_{\delta}(g P)}$.

Choose $i \in\{0,1,2\}$ so that $g P \neq g_{i} P_{i}$. Since $c_{i}^{\prime} \subseteq \mathcal{N}_{\delta}\left(g_{i} P_{i}\right)$, it follows from Theorem 5.2 that $c_{i}^{\prime}$ has length less than $\kappa=\kappa(2 \delta)$. By Theorem 5.5, the endpoints $z_{j}$ and $z_{k}$ of $c_{i}^{\prime}$ are within a distance $\mu$ of the segments $c_{j}$ and $c_{k}$ respectively. Thus we have

$$
d\left(z_{j}, c_{i}\right)=0, \quad d\left(z_{j}, c_{j}\right)<\mu \quad \text { and } \quad d\left(z_{j}, c_{k}\right)<\kappa+\mu .
$$

In other words, the ball of radius $\kappa+\mu$ centered at $z_{j}$ intersects all three sides of $\Delta$. It follows that, in all cases, there exists a point $z$ such that the $\delta^{\prime}$-ball centered at $z$ intersects all three sides of $\Delta$, where $\delta^{\prime}=\delta+\kappa+\mu$. Now for each choice of $\{j, k\}=\{1,2\}$ we have

and

$$
z \in \mathcal{N}_{\delta^{\prime}+\tau}\left(g_{0} P_{0}\right) \cap \mathcal{N}_{\delta^{\prime}+\tau}\left(g_{j} P_{j}\right)
$$

$$
y_{k} \in \mathcal{N}_{\tau}\left(g_{0} P_{0}\right) \cap \mathcal{N}_{\tau}\left(g_{j} P_{j}\right) .
$$

Consequently, Theorem 5.2 gives an upper bound $\kappa^{\prime}:=\kappa\left(\delta^{\prime}+\tau\right)$ on the distance $d\left(y_{k}, z\right)$. Varying $k \in\{1,2\}$, we see that $d\left(y_{1}, y_{2}\right)$ is less than $2 \kappa^{\prime}$. 
The following result is analogous to the previous lemma, but for quadrilaterals instead of triangles. If each side of a quadrilateral lies close to a peripheral coset, then either all four cosets are equal or some pair of vertices of the quadrilateral is close together.

Lemma 5.7 Let $(G, \mathbb{P})$ be relatively hyperbolic. For each $\eta>0$, there is a constant $\xi>0$ such that the following holds. Let $g_{0} P_{0}, g_{1} P_{1}, g_{2} P_{2}$ and $g_{3} P_{3}$ be peripheral cosets. Suppose for each $i \in\{0,1,2,3\}$ there is a point

$$
\left.y_{i} \in \mathcal{N}_{\eta}\left(g_{i-1} P_{i-1}\right) \cap \mathcal{N}_{\eta}\left(g_{i} P_{i}\right) \quad \text { (indices modulo } 4\right) .
$$

If the pairwise distances between the points $\left\{y_{0}, y_{1}, y_{2}, y_{3}\right\}$ are all at least $\xi$, then all four peripheral cosets are equal.

Proof Suppose the four peripheral cosets are not all equal. We will show that one of the pairwise distances between the $y_{i}$ is bounded above by an appropriate constant $\xi$. For each $i$, let $c_{i}$ be a geodesic in $X$ from $y_{i}$ to $y_{i+1}$. Then by Theorem 5.3, we have

$$
c_{i} \subseteq \mathcal{N}_{\tau}\left(g_{i} P_{i}\right)
$$

for some $\tau$ depending on $\eta$.

If $g_{i} P_{i}=g_{i+1} P_{i+1}$ for some $i$, the result follows immediately from Lemma 5.6. Now suppose $g_{i} P_{i}=g_{i+2} P_{i+2}$ for some $i$, but $g_{i+1} P_{i+1} \neq g_{i} P_{i}$. Then $c_{i+1}$ lies in the $\tau$-neighborhood of both $g_{i} P_{i}$ and $g_{i+1} P_{i+1}$. But then Theorem 5.2 gives an upper bound $\kappa_{0}:=\kappa(\tau)$ on $d\left(y_{i+1}, y_{i+2}\right)$, completing the proof. Thus it suffices to assume that the four peripheral cosets are all different.

By hypothesis, the points $y_{0}$ and $y_{1}$ lie in $\mathcal{N}_{\eta}\left(g_{0} P_{0}\right)$. Let $u$ be the point where $c_{3}$ first enters the closed $\eta$-neighborhood of $g_{0} P_{0}$ when traversed from $y_{3}$ to $y_{0}$. Similarly, let $v$ be the point where $c_{1}$ first enters the closed $\eta$-neighborhood of $g_{0} P_{0}$ when traversed from $y_{2}$ to $y_{1}$. Since $g_{0} P_{0} \notin\left\{g_{1} P_{1}, g_{3} P_{3}\right\}$, Theorem 5.2 gives an upper bound $\kappa_{1}:=\kappa(\eta)$ on the distances $d\left(y_{0}, u\right)$ and $d\left(y_{1}, v\right)$. We may assume without loss of generality that $\eta$ is greater than the constant $v_{0}$ given by Proposition 5.4. Since $g_{0} P_{0} \neq g_{2} P_{2}$, Proposition 5.4 gives an upper bound $D_{1}=D_{1}(\eta)$ on the distance $d(u, v)$. Thus we have

$$
d\left(y_{0}, y_{1}\right)<D_{1}+2 \kappa_{1}
$$

completing the proof of the lemma. 


\section{The geometry of relative Cayley graphs}

The results in this section and the next involve the interplay between two different metrics on a relatively hyperbolic $G$ with a fixed finite generating set $\mathcal{S}$. When a metric is not specified, all distances are assumed to refer to the word metric $d_{\mathcal{S}}$ or (equivalently) in the Cayley graph $\operatorname{Cayley}\left(G, d_{\mathcal{S}}\right)$. Let $\mathcal{P}$ be the union of all the peripheral subgroups of $G$. We will also use the word metric $d_{\mathcal{S} \cup \mathcal{P}}$ and the Cayley $\operatorname{graph} \operatorname{Cayley}(G, \mathcal{S} \cup \mathcal{P})$ for the (typically infinite) generating set $\mathcal{S} \cup \mathcal{P}$. Whenever we use this "relative metric" or "relative Cayley graph," we will explicitly call attention to its use. In particular, if $A \subset G$ the notation $\mathcal{N}_{\epsilon}(A)$ always refers to the $\epsilon$-neighborhood of $A$ using the metric $d_{\mathcal{S}}$, and the notation $B(x, \epsilon)$ refers to an $\mathcal{S}$-metric ball.

Geodesics in the relative Cayley graph are sometimes referred to as relative geodesics in $G$, etc. Observe that Cayley $(G, \mathcal{S})$ is a subgraph of Cayley $(G, \mathcal{S} \cup \mathcal{P})$ containing all the vertices but omitting the edges labelled by elements of $\mathcal{P}$.

The next result is a relatively hyperbolic analogue of the Morse Lemma, dealing with a pair of relative geodesics whose respective endpoints are close together in $d_{\mathcal{S}}$.

Proposition 6.1 (Osin [15, Proposition 3.15]) Let $(G, \mathbb{P})$ be relatively hyperbolic. For each $k \geq 0$, there is a constant $\rho=\rho(k)$ such that the following holds. Let $c$ and $c^{\prime}$ be relative geodesics in Cayley $(G, \mathcal{S} \cup \mathcal{P})$ such that their initial endpoints are within an $\mathcal{S}$-distance $k$ and their terminal endpoints are also within an $\mathcal{S}$-distance $k$. Then the set of vertices of $c$ and the set of vertices of $c^{\prime}$ are within a Hausdorff $\mathcal{S}$-distance $\rho$.

The following result is an analogue of Theorem 5.5 for relative geodesic triangles.

Theorem 6.2 (Osin $[15$, Theorem 3.26]) Let $(G, \mathbb{P})$ be relatively hyperbolic. Then there is a constant $v>0$ such that if $c_{0} \cup c_{1} \cup c_{2}$ is a relative geodesic triangle in Cayley $(G, \mathcal{S} \cup \mathcal{P})$ then for each vertex $v$ of $c_{0}$ there is a vertex $u$ in the union $c_{1} \cup c_{2}$ such that

$$
d_{\mathcal{S}}(u, v)<v .
$$

Subdividing an $n$-gon into $n-2$ triangles and applying the previous theorem, we get the following corollary. As an aside we note that the linear function $n-2$ in the conclusion of the corollary can be improved to a logarithmic function of $n$ by subdividing more carefully.

Corollary 6.3 Let $(G, \mathbb{P})$ and $v$ be as in the previous theorem. If $c_{1} \cup \cdots \cup c_{n}$ is a relative geodesic $n$-gon in Cayley $(G, \mathcal{S} \cup \mathcal{P})$, then for each vertex $v$ on $c_{1}$ there is a vertex $u$ on the union $c_{2} \cup \cdots \cup c_{n}$ such that

$$
d_{\mathcal{S}}(u, v)<(n-2) v .
$$


The notion of saturation of a quasigeodesic was introduced by Druț-Sapir [5]. The saturation of an arbitrary subset is defined similarly, as follows.

Definition 6.4 Suppose $(G, \mathbb{P})$ is relatively hyperbolic, and let $Y$ be a subset of $G$. For each $v>0$ the $v$-saturation of $Y$, denoted $\operatorname{Sat}_{v}(Y)$, is the union of $Y$ and every peripheral coset intersecting $\mathcal{N}_{v}(Y)$.

We conclude the section with a mild generalization of Proposition 6.1.

Proposition 6.5 Let $(G, \mathbb{P})$ be relatively hyperbolic. For each $\tau>0$ there is a constant $\lambda=\lambda(\tau)>0$ such that the following holds. Let $c$ and $c^{\prime}$ be relative geodesic segments in $\operatorname{Cayley}(G, \mathcal{S} \cup \mathcal{P})$, such that the endpoints $x_{0}$ and $x_{1}$ of $c^{\prime}$ lie within an $\mathcal{S}$-distance $\tau$ of $\operatorname{Sat}_{\tau}(\operatorname{Vert}(c))$. Let $v$ be a vertex of $c^{\prime}$. Then $v$ lies within an $\mathcal{S}$-distance $\lambda$ of either $x_{0}, x_{1}$, or a vertex of $c$.

Proof For each $i \in\{0,1\}$, we define a path $c_{i}$ in $\operatorname{Cayley}(G, \mathcal{S} \cup \mathcal{P})$ from $x_{i}$ to $c$ as follows. If $x_{i}$ is within an $\mathcal{S}$-distance $\tau$ of a vertex of $c$, then let $c_{i}$ be an $\mathcal{S}$-geodesic of shortest length from $x_{i}$ to $c$. Otherwise, $x_{i}$ is within an $\mathcal{S}$-distance $\tau$ of a left coset $g_{i} P_{i}$ that is within an $\mathcal{S}$-distance $\tau$ of a vertex of $c$. In the latter case, let $c_{i}$ be the concatenation of an $\mathcal{S}$-geodesic $q_{i}$ of shortest length from $x_{i}$ to $g_{i} P_{i}$ followed by a peripheral edge $e_{i}$ in $g_{i} P_{i}$ followed by an $\mathcal{S}$-geodesic $r_{i}$ of shortest length from $g_{i} P_{i}$ to a vertex of $c$. Let $a_{i} \in C$ denote the terminal vertex of $c_{i}$, and let $\bar{c}$ denote the portion of $c$ from $a_{0}$ to $a_{1}$.

Note that $c_{i}$ is a concatenation of at most $2 \tau+1$ edges, each of which is a relative geodesic of length one. Thus $c^{\prime} \cup c_{0} \cup \bar{c} \cup c_{1}$ is a relative geodesic polygon with at most $4 \tau+4$ sides. Consequently, by Corollary 6.3 each vertex of $c^{\prime}$ is within an $\mathcal{S}$-distance $(4 \tau+2) v$ of some vertex of $c_{0} \cup \bar{c} \cup c_{1}$.

Choose a vertex $v$ of $c^{\prime}$ such that $v$ is at an $\mathcal{S}$-distance more than $(4 \tau+2) v+\tau$ from $x_{0}$. If $c_{0}$ is an $\mathcal{S}$-geodesic of length at most $\tau$, then, by the triangle inequality, no vertex of $c_{0}$ can be within an $\mathcal{S}$-distance $(4 \tau+2) v$ of $v$. Thus $v$ is within an $\mathcal{S}-$ distance $(4 \tau+2) v$ of some vertex of $\bar{c} \cup c_{1}$. On the other hand, if $c_{0}$ is a concatenation $q_{0} \cup e_{0} \cup r_{0}$ as described above, then $q_{0}$ has $\mathcal{S}$-length at most $\tau$. By a similar argument, it follows that $v$ is within an $\mathcal{S}$-distance $(4 \tau+2) v$ of some vertex of $r_{0} \cup \bar{c} \cup c_{1}$. Since $r_{0}$ has $\mathcal{S}$-length at most $\tau$, each of its vertices lies within an $\mathcal{S}$-distance $\tau$ of the vertex $a_{0}$. Thus in either case, $v$ is within an $\mathcal{S}$-distance $(4 \tau+2) v+\tau$ of some vertex of $\bar{c} \cup c_{1}$.

Interchanging the roles of $c_{0}$ and $c_{1}$, we see that if $v$ is also at least an $\mathcal{S}$-distance $(4 \tau+2) v+\tau$ from $x_{1}$ then it is within an $\mathcal{S}$-distance $(4 \tau+2) v+\tau$ from some vertex of $\bar{c}$. Setting $\lambda=(4 \tau+2) v+\tau$ completes the proof. 
Definition 6.6 Let $(G, \mathbb{P})$ be relatively hyperbolic with a finite generating set $\mathcal{S}$. Let $c$ be a geodesic of Cayley $(G, \mathcal{S})$ and let $\epsilon$ and $R$ be positive constants. A point $x \in c$ is $(\epsilon, R)$-deep in a peripheral coset $g P$ (with respect to $c$ ) if $x$ is not within an $\mathcal{S}$-distance $R$ of an endpoint of $c$ and if $B(x, R) \cap c$ lies in $\mathcal{N}_{\epsilon}(g P)$. If $x$ is not $(\epsilon, R)$-deep in any $g P$ then $x$ is an $(\epsilon, R)$-transition point of $c$.

Proposition 6.7 (Hruska [10, Proposition 8.13]) Let $(G, \mathbb{P})$ be a relatively hyperbolic group with a finite generating set $\mathcal{S}$. There exist constants $\epsilon, R$ and $L$ such that the following holds. Let $c$ be any geodesic of Cayley $(G, \mathcal{S})$ with endpoints in $G$, and let $c^{\prime}$ be a relative geodesic in $\operatorname{Cayley}(G, \mathcal{S} \cup \mathcal{P})$ with the same endpoints as $c$. Then the set of vertices of $c^{\prime}$ is at a Hausdorff $\mathcal{S}$-distance at most $L$ from the set of $(\epsilon, R)$-transition points of $c$.

Furthermore, let $\bar{c}$ be a connected component of the set of all $(\epsilon, R)$-deep points of $c$. Then there is a peripheral coset $g P$ such that each $x \in \bar{c}$ is $(\epsilon, R)$-deep in $g P$ and is not $(\epsilon, R)$-deep in any other peripheral coset.

\section{Relatively quasiconvex subspaces}

In the theory of word hyperbolic groups, the most natural subgroups (and subspaces) are the quasiconvex subgroups (and subspaces). In this section, we examine "relatively quasiconvex" subspaces, which play an analogous role in the theory of relatively hyperbolic groups. The definition of relative quasiconvexity given below was introduced by Osin [15].

Definition 7.1 Let $(G, \mathbb{P})$ be relatively hyperbolic such that $G$ is generated by a finite set $\mathcal{S}$. For $\sigma \geq 0$, a subset $A \leq G$ is $\sigma$-relatively quasiconvex in $G$ if the following condition holds: Let $c$ be a relative geodesic in Cayley $(G, \mathcal{S} \cup \mathcal{P})$ connecting two points of $Y$. Then each vertex $v$ of $c$ lies in the $\sigma$-neighborhood of $A$.

A subset $A \subseteq G$ is relatively quasiconvex if it is $\sigma$-relatively quasiconvex for some $\sigma \geq 0$.

Proposition 7.2 (Osin [15, Proposition 4.10]) Relative quasiconvexity of a subset $A \subseteq G$ is independent of the choice of finite generating set $\mathcal{S}$ for $G$.

The following result analogous to Theorem 5.5 follows immediately from the definition of relative quasiconvexity.

Proposition 7.3 Each peripheral coset $g P$ in a relatively hyperbolic group is 0relatively quasiconvex. 
The following result was proved independently by Hruska [10, Theorem 9.1] and Martinez-Pedroza [12, Proposition 1.5].

Proposition 7.4 Let $(G, \mathbb{P})$ be relatively hyperbolic and let $H \leq G$ be $\sigma$-relatively quasiconvex. The infinite maximal parabolic subgroups $Q=H \cap \mathrm{gPg}^{-1}$ of $H$ lie in finitely many $H$-conjugacy classes, and the corresponding peripheral cosets $g P$ in $G$ lie in finitely many $H$-orbits.

Proposition 7.5 Let $(G, \mathbb{P})$ be relatively hyperbolic. Choose positive constants $\sigma$ and $\tau$. Then there is a constant $R=R(\sigma, \tau)$ such that the following holds. Choose $n \geq 3$, and let $\mathcal{A}=\left\{A_{1}, \ldots, A_{n}\right\}$ be a collection of $\sigma$-relatively quasiconvex subsets of $G$. Suppose for each $i=1, \ldots, n$ there is a point

$$
x_{i} \in \bigcap\left\{\mathcal{N}_{\tau}\left(A_{j}\right) \mid 1 \leq j \leq n, j \neq i\right\} .
$$

Then we have the following consequences:

(1) There exists a vertex

$$
x_{0} \in \mathcal{N}_{R}\left(\operatorname{Sat}_{R}\left(A_{1}\right)\right) \cap \mathcal{N}_{R}\left(A_{2}\right) \cap \cdots \cap \mathcal{N}_{R}\left(A_{n}\right) .
$$

(2) If $A_{1}$ is a left coset of a peripheral subgroup, then there exists a vertex

$$
x_{0} \in \mathcal{N}_{R}\left(A_{1}\right) \cap \cdots \cap \mathcal{N}_{R}\left(A_{n}\right) .
$$

(3) If $A_{0}$ is another $\sigma$-relatively quasiconvex subset of $G$ such that $x_{i}$ is contained in $\mathcal{N}_{\tau}\left(\operatorname{Sat}_{\tau}\left(A_{0}\right)\right)$ for all $i=1, \ldots, n$, then there exists a vertex $x_{0}$ satisfying (2) such that $x_{0} \in \mathcal{N}_{R}\left(\operatorname{Sat}_{R}\left(A_{0}\right)\right)$.

Proof Choose relative geodesics

$$
c_{1}=\left[x_{2}, x_{3}\right], \quad c_{2}=\left[x_{3}, x_{1}\right] \quad \text { and } \quad c_{3}=\left[x_{1}, x_{2}\right]
$$

in Cayley $(G, \mathcal{S} \cup \mathcal{P})$, and consider the triangle $\Delta:=c_{1} \cup c_{2} \cup c_{3}$. By Proposition 6.1 and the $\sigma$-relative quasiconvexity of $A_{\ell}$, there is a constant $\rho=\rho(\tau)$ such that if $i \in\{1,2,3\}$ and $\ell \in\{i\} \cup\{4, \ldots, n\}$ then each vertex of $c_{i}$ lies within an $\mathcal{S}$-distance $\sigma+\rho$ of $A_{\ell}$.

By Theorem 6.2, there is a constant $v$ such that for each choice of $\{i, j, k\}=\{1,2,3\}$ each vertex of $c_{i}$ lies within an $\mathcal{S}$-distance $v$ of a vertex of $c_{j} \cup c_{k}$. It follows that $c_{i}$ contains a pair of adjacent vertices $v_{i j}$ and $v_{i k}$ connected by an edge $e_{i}$ such that $v_{i j}$ is within an $\mathcal{S}$-distance $v$ of a vertex of $c_{j}$ and $v_{i k}$ is within an $\mathcal{S}$-distance $v$ of a vertex of $c_{k}$. 
If for some choice of $\{i, j, k\}=\{1,2,3\}$, the edge $e_{i}$ is labelled by a generator in $\mathcal{S}$, then for all $\ell \in\{i\} \cup\{4, \ldots, n\}$ we have:

$$
v_{i j} \in \mathcal{N}_{1+v+\sigma+\rho}\left(A_{k}\right) \cap \mathcal{N}_{\nu+\sigma+\rho}\left(A_{j}\right) \cap \mathcal{N}_{\sigma+\rho}\left(A_{\ell}\right)
$$

If not, then $e_{3}$ is labelled by a generator in $\mathcal{P}$ and $v_{32}$ and $v_{31}$ are vertices of a peripheral coset $g P$. Since $d_{\mathcal{S}}\left(v_{31}, A_{1}\right)<v+\sigma+\rho$, the coset $g P$ is contained in $\operatorname{Sat}_{v+\sigma+\rho}\left(A_{1}\right)$. Whenever $\ell=3, \ldots, n$, we now have:

$$
v_{32} \in \operatorname{Sat}_{v+\sigma+\rho}\left(A_{1}\right) \cap \mathcal{N}_{v+\sigma+\rho}\left(A_{2}\right) \cap \mathcal{N}_{\sigma+\rho}\left(A_{\ell}\right)
$$

In either case, choosing $x_{0}:=v_{32}$ and $R \geq 1+v+\sigma+\rho$ completes the proof of (1).

Now let us consider assertion (2). Suppose the subspace $A_{1}$ is a peripheral coset $g P$. If for some $i \in\{1,2,3\}$ the edge $e_{i}$ corresponds to a generator in $\mathcal{S}$ then we are done, since (7-1) shows that $v_{i j}$ is within an $\mathcal{S}$-distance $1+v+\sigma+\rho$ of $A_{\ell}$ for all $\ell=1, \ldots, n$.

It therefore suffices to assume that each $e_{i}$ corresponds to a generator from $\mathcal{P}$ such that $v_{i j}, v_{i k} \in g_{i} P_{i}$. for some peripheral coset $g_{i} P_{i}$. If $g P \neq g_{1} P_{1}$, then by Theorem 5.2, the $\mathcal{S}$-distance between $v_{12}$ and $v_{13}$ is at most $\kappa=\kappa(\sigma+\rho)$. In this case, a computation similar to (7-1) shows that $v_{12}$ is within an $\mathcal{S}$-distance $\kappa+v+\sigma+\rho$ of $A_{\ell}$ for all $\ell=1, \ldots, n$. Similarly if the cosets $g_{1} P_{1}, g_{2} P_{2}$ and $g_{3} P_{3}$ are not all the same, then by Lemma 5.6 the $\mathcal{S}$-distance between some pair $v_{i j}$ and $v_{i k}$ is bounded above by $\zeta=\zeta(v)$. Therefore $v_{i j}$ is within an $\mathcal{S}$-distance $\zeta+v+\sigma+\rho$ of $A_{\ell}$ for all $\ell=1, \ldots, n$.

The only remaining possibility is that the four cosets $g P, g_{1} P_{1}, g_{2} P_{2}$ and $g_{3} P_{3}$ are all equal. In particular, we have $A_{1}=g P=g_{3} P_{3}$. It follows that

$$
d_{\mathcal{S}}\left(v_{32}, A_{1}\right)=0, \quad d_{\mathcal{S}}\left(v_{32}, A_{2}\right)<v+\sigma+\rho \quad \text { and } \quad d_{\mathcal{S}}\left(v_{32}, A_{\ell}\right)<\sigma+\rho
$$

for all $\ell=3, \ldots, n$. In all possible cases, to complete the proof of (2) it suffices to choose

$$
R>1+\kappa+\zeta+v+\sigma+\rho .
$$

Finally, we turn our attention to assertion (3). We have shown that assertions (1) and (2) hold for sufficiently large $R$ and for some vertex $x_{0}$ lying on one of the sides $c_{i}$ of the triangle $\Delta$. Suppose the endpoints $x_{j}$ and $x_{k}$ of $c_{i}$ lie within an $\mathcal{S}$-distance $\tau$ of $\operatorname{Sat}_{\tau}\left(A_{0}\right)$. Choose points $u$ and $v$ in $A_{0}$ such that $x_{j} \in \mathcal{N}_{\tau}\left(\operatorname{Sat}_{\tau}(u)\right)$ and $x_{k} \in \mathcal{N}_{\tau}\left(\operatorname{Sat}_{\tau}(v)\right)$, and let $\bar{c}$ be a geodesic in $\operatorname{Cayley}(G, \mathcal{S} \cup \mathcal{P})$ from $u$ to $v$.

It follows from Proposition 6.5 that the vertex $x_{0}$ of $c_{i}$ lies within a distance $\lambda=\lambda(\tau)$ of either $x_{j}, x_{k}$, or a vertex of $\bar{c}$. Since $A_{0}$ is $\sigma$-quasiconvex, it follows that $x_{0}$ lies 
within a distance $\lambda+\sigma$ of $\left\{x_{j}, x_{k}\right\} \cup A_{0}$. In particular, $x_{0}$ is within an $\mathcal{S}$-distance $\lambda+\sigma+\tau$ of $\operatorname{Sat}_{\tau}\left(A_{0}\right)$, establishing (3) for all $R>\lambda+\sigma+\tau$.

Corollary 7.6 Let $(G, \mathbb{P})$ be relatively hyperbolic. Choose positive constants $\sigma$ and $D$ and an integer $n \geq 1$. There is a constant $R_{1}=R_{1}(\sigma, D, n)$ such that the following holds. Let $\mathcal{A}=\left\{A_{1}, \ldots, A_{n}\right\}$ be a set of $\sigma$-relatively quasiconvex subsets of $G$. Suppose the following intersection is nonempty:

$$
\bigcap_{i=1}^{n} \mathcal{N}_{D}\left(A_{i}\right)
$$

If there is a peripheral coset $g P$ such that for all $i$ the intersection $\mathcal{N}_{D}\left(A_{i}\right) \cap \mathcal{N}_{D}(g P)$ is nonempty, then the following intersection is nonempty:

$$
\mathcal{N}_{R_{1}}(g P) \cap \mathcal{N}_{R_{1}}\left(A_{1}\right) \cap \cdots \cap \mathcal{N}_{R_{1}}\left(A_{n}\right)
$$

Proof Recall that $g P$ is 0 -relatively hyperbolic. When $n=1$, the result is trivial. We proceed by induction on $n$ for $n \geq 2$. Let $\tau:=R_{1}(\sigma, D, n-1)$ denote the constant given by the inductive hypothesis. By assumption, $\bigcap_{i=1}^{n} \mathcal{N}_{D}\left(A_{i}\right)$ is nonempty. By induction, for each $i=1, \ldots, n$ the following intersection is nonempty:

$$
\mathcal{N}_{\tau}(g P) \cap \bigcap\left\{\mathcal{N}_{\tau}\left(A_{j}\right) \mid 1 \leq j \leq n, j \neq i\right\}
$$

Applying Proposition 7.5(2) to the collection $\left\{g P, A_{1}, \ldots, A_{n}\right\}$ of $\sigma$-quasiconvex subspaces, the result now follows.

Proposition 7.7 Let $(G, \mathbb{P})$ be relatively hyperbolic. Choose positive constants $\sigma$ and $D$ and an integer $n \geq 1$. Let $\mathcal{A}$ be an arbitrary set of $\sigma$-relatively quasiconvex subsets of $G$ such that for each $A, A^{\prime} \in \mathcal{A}$ we have $d_{\mathcal{S}}\left(A, A^{\prime}\right)<D$. Then there is a constant $M_{0}=M_{0}(\sigma, D, n)$ so that whenever $M \geq M_{0}$ at least one of the following holds.

(1) For every subset $\left\{A_{1}, \ldots, A_{n}\right\} \subseteq \mathcal{A}$, the following intersection is nonempty:

$$
\bigcap_{\ell=1}^{n} \mathcal{N}_{M}\left(A_{\ell}\right)
$$

(2) There is a peripheral coset $g P$ such that $d_{\mathcal{S}}(g P, A)<M$ for all $A \in \mathcal{A}$. Furthermore, for all $A, A^{\prime} \in \mathcal{A}$, the following triple intersection is nonempty:

$$
\mathcal{N}_{M}(g P) \cap \mathcal{N}_{M}(A) \cap \mathcal{N}_{M}\left(A^{\prime}\right)
$$


Proof Recall that every peripheral coset $g P$ is 0 -quasiconvex, as observed in Proposition 7.3. In order to establish (2), it is sufficient to find a constant $M$ and a peripheral coset $g P$ such that $d_{\mathcal{S}}(g P, A)<M$ for all $A \in \mathcal{A}$. For then applying Proposition 7.5 (2) to each collection $\left\{g P, A, A^{\prime}\right\}$ with $A, A^{\prime} \in \mathcal{A}$ gives a larger constant $M^{\prime}$ so that the following triple intersection is nonempty:

$$
\mathcal{N}_{M^{\prime}}(g P) \cap \mathcal{N}_{M^{\prime}}(A) \cap \mathcal{N}_{M^{\prime}}\left(A^{\prime}\right)
$$

The proposition is trivial when $n \leq 2$. We proceed by induction on $n$ for $n \geq 3$. Let $\tau$ denote the constant $M_{0}(\sigma, D, n-1)$ given by the inductive hypothesis. We will show that the proposition holds whenever $M$ is sufficiently large (in terms of $\sigma$ and $\tau$ ).

Suppose $\left\{A_{0}, \ldots, A_{n-1}\right\}$ is a subset of $\mathcal{A}$ such that

$$
\bigcap_{\ell=0}^{n-1} \mathcal{N}_{M}\left(A_{\ell}\right)=\varnothing .
$$

If no such set exists, there is nothing to prove. Furthermore, if there is a peripheral coset $g P$ such that $d_{\mathcal{S}}(P, A)<v$ for all $A \in \mathcal{A}$, then we are done. Thus we may assume that for every collection of $n-1$ distinct elements of $\mathcal{A}$, their $v$-neighborhoods have a nonempty intersection.

Applying Proposition 7.5(1) to the collection $\left\{A_{0}, \ldots, A_{n-1}\right\}$ gives $R_{0}=R_{0}(\sigma, \tau)$ and a point

$$
x_{n} \in \mathcal{N}_{R_{0}}\left(\operatorname{Sat}_{R_{0}}\left(A_{0}\right)\right) \cap \mathcal{N}_{R_{0}}\left(A_{1}\right) \cap \cdots \cap \mathcal{N}_{R_{0}}\left(A_{n-1}\right) .
$$

If we choose $M$ greater than $R_{0}$, then (7-2) implies that $d_{\mathcal{S}}\left(x_{n}, A_{0}\right)$ is at least $R_{0}$. Thus $x_{n} \in \mathcal{N}_{R_{0}}\left(g_{0} P_{0}\right)$ for some peripheral coset $g_{0} P_{0}$ such that $g_{0} P_{0} \subseteq \operatorname{Sat}_{R_{0}}\left(A_{0}\right)$. Note that $d_{\mathcal{S}}\left(g_{0} P_{0}, A_{\ell}\right)<2 R_{0}$ for all $\ell=0, \ldots, n-1$. If $\mathcal{A}=\left\{A_{0}, \ldots, A_{n-1}\right\}$, we are done. Otherwise, choose an arbitrary $A_{n} \in \mathcal{A}-\left\{A_{0}, \ldots, A_{n-1}\right\}$. To complete the proof, we will bound the $\mathcal{S}$-distance from $A_{n}$ to $g_{0} P_{0}$ in terms of $\sigma$ and $\tau$, provided that $M$ is sufficiently large.

For each $i=1, \ldots, n-1$, applying Proposition 7.5(1) to the collection

$$
\left\{A_{0}, \ldots, A_{i-1}, A_{i+1}, \ldots, A_{n}\right\}
$$

gives a point

$x_{i} \in \mathcal{N}_{R_{0}}\left(\operatorname{Sat}_{R_{0}}\left(A_{0}\right)\right) \cap \mathcal{N}_{R_{0}}\left(A_{1}\right) \cap \cdots \cap \mathcal{N}_{R_{0}}\left(A_{i-1}\right) \cap \mathcal{N}_{R_{0}}\left(A_{i+1}\right) \cap \cdots \cap \mathcal{N}_{R_{0}}\left(A_{n}\right)$.

Notice that the points $x_{1}, \ldots, x_{n}$ and subspaces $A_{0}, A_{1}, \ldots, A_{n}$ satisfy the hypothesis of Proposition 7.5(3). Therefore there exist a constant $R_{1}:=R\left(\sigma, R_{0}\right)$ and a point

$$
x_{0} \in \mathcal{N}_{R_{1}}\left(\operatorname{Sat}_{R_{1}}\left(A_{0}\right)\right) \cap \mathcal{N}_{R_{1}}\left(A_{1}\right) \cap \cdots \cap \mathcal{N}_{R_{1}}\left(A_{n}\right) .
$$


Choose a relative geodesic $c$ from $x_{0}$ to $x_{n}$. Since the endpoints of $c$ lie within a distance $R_{0}+R_{1}$ of $A_{\ell}$ for each $\ell=1, \ldots, n-1$, we know from Proposition 6.1 and the $\sigma$-relative quasiconvexity of $A_{\ell}$ that each vertex of $c$ lies within an $\mathcal{S}$-distance $\sigma+\rho$ of $A_{\ell}$, where $\rho=\rho\left(R_{0}+R_{1}\right)$. However, by (7-2) there is no vertex within a distance $M$ of every $A_{\ell}$ for $\ell=0, \ldots, n$.

Roughly speaking, it follows that once $M$ is sufficiently large, each vertex of $c$ will be far from $A_{0}$. We have already used this principle to conclude that $x_{n}$ is within an $\mathcal{S}$-distance $R_{0}$ of $g_{0} P_{0}$, which is itself within an $\mathcal{S}$-distance $R_{0}$ of $A_{0}$, once $M$ is sufficiently large. Let $y_{n}$ be a vertex of $A_{0}$ within an $\mathcal{S}$-distance $R_{0}$ of $g_{0} P_{0}$. A similar argument shows that $x_{0}$ is within a distance $R_{1}$ of a peripheral coset $g_{1} P_{1}$, which is itself within a distance $R_{1}$ of $A_{0}$, for sufficiently large $M$. Let $y_{0}$ be a vertex of $A_{0}$ within an $\mathcal{S}$-distance $R_{1}$ of $g_{1} P_{1}$. Choose a relative geodesic $\bar{c}$ of Cayley $(G, \mathcal{S} \cup \mathcal{P})$ from $y_{0}$ to $y_{n}$.

Since $x_{0}$ and $x_{n}$ are both contained in $\mathcal{N}_{R_{0}+R_{1}}\left(\operatorname{Sat}_{R_{0}+R_{1}}(\bar{c})\right)$, it follows from Proposition 6.5 that each vertex of $c$ lies within an $\mathcal{S}$-distance $\lambda$ of either $x_{0}, x_{n}$, or a vertex of $\bar{c}$. By the $\sigma$-relative quasiconvexity of $A_{0}$, each vertex of $\bar{c}$ lies within an $\mathcal{S}$-distance $\sigma$ of $A_{0}$. Thus each vertex of $c$ either lies within an $\mathcal{S}$-distance $\lambda$ of $\left\{x_{0}, x_{n}\right\}$ or lies within an $\mathcal{S}$-distance $\lambda+\sigma$ of $A_{0}$ for some $\lambda=\lambda\left(R_{0}+R_{1}\right)$. Suppose $v$ is a vertex of $c$ whose $\mathcal{S}$-distance from $A_{0}$ is less than $\lambda+\sigma$. Once $M>\lambda+\sigma+\rho$, the existence of such a vertex $v$ contradicts (7-2). Therefore we may assume that each vertex $v$ of $c$ lies within an $\mathcal{S}$-distance $\lambda$ of $\left\{x_{0}, x_{n}\right\}$. A similar argument shows that each vertex $v$ of $\bar{c}$ lies within an $\mathcal{S}$-distance $\lambda$ of $\left\{y_{0}, y_{n}\right\}$, since otherwise $v$ would be close to both $A_{0}$ and a vertex of $c$, contradicting (7-2) once $M$ is sufficiently large.

Let $e$ be an edge of $c$ with endpoints $v_{0}$ and $v_{n}$ such that $v_{0}$ is within an $\mathcal{S}$-distance $\lambda$ of $x_{0}$ and $v_{n}$ is within an $\mathcal{S}$-distance $\lambda$ of $x_{n}$. If $e$ corresponds to a generator in $\mathcal{S}$, then $d_{\mathcal{S}}\left(x_{0}, x_{n}\right)<2 \lambda+1$. Since $d_{\mathcal{S}}\left(x_{n}, g_{0} P_{0}\right)<R_{0}$ and $d_{\mathcal{S}}\left(x_{0}, A_{n}\right)<R_{1}$, it follows that $d_{\mathcal{S}}\left(A_{n}, g_{0} P_{0}\right)<2 \lambda+1+R_{0}+R_{1}$ and we are done. Thus we may assume that $e$ corresponds to a generator in $\mathcal{P}$ and that $v_{0}, v_{n} \in g_{2} P_{2}$ for some peripheral coset $g_{2} P_{2}$.

Now let $\bar{e}$ be an edge of $\bar{c}$ with endpoints $w_{0}$ and $w_{n}$ within a distance $\lambda$ of $y_{0}$ and $y_{n}$ respectively. We will see that $\bar{e}$ must also correspond to a generator in $\mathcal{P}$. Suppose instead that $\bar{e}$ corresponds to a generator in $\mathcal{S}$. Then $d_{\mathcal{S}}\left(y_{0}, y_{n}\right)$ is less than $2 \lambda+1$. In this case, consider the three peripheral cosets $g_{0} P_{0}, g_{1} P_{1}$, and $g_{2} P_{2}$. Recall that $x_{0}$ lies within a distance $R_{1}+\lambda$ of both $g_{1} P_{1}$ and $g_{2} P_{2}$. If $g_{0} P_{0} \in\left\{g_{1} P_{1}, g_{2} P_{2}\right\}$, then $d\left(x_{0}, g_{0} P_{0}\right)<R_{1}+\lambda$, completing the proof as before since $d\left(x_{0}, A_{n}\right)<R_{0}$. On the other hand, if $g_{0} P_{0} \notin\left\{g_{1} P_{1}, g_{2} P_{2}\right\}$, we can apply Lemma 5.6 to $g_{0} P_{0}, g_{1} P_{1}$ and $g_{2} P_{2}$ to produce an upper bound $\zeta$ on the distance from $x_{n}$ to $A_{0}$, contradicting (7-2) 
once $M$ is at least $\zeta+\sigma+\rho$ as before. Therefore we may assume that $\bar{e}$ corresponds to a generator in $\mathcal{P}$ and that $w_{0}, w_{n} \in g_{3} P_{3}$ for some peripheral coset $g_{3} P_{3}$.

We now have a cycle of four peripheral cosets $g_{0} P_{0}, g_{2} P_{2}, g_{1} P_{1}$, and $g_{3} P_{3}$ such that the following hold for $\eta:=R_{0}+R_{1}+\lambda$ :

and

$$
\begin{aligned}
& x_{n} \in \mathcal{N}_{\eta}\left(g_{0} P_{0}\right) \cap \mathcal{N}_{\eta}\left(g_{2} P_{2}\right) \\
& x_{0} \in \mathcal{N}_{\eta}\left(g_{2} P_{2}\right) \cap \mathcal{N}_{\eta}\left(g_{1} P_{1}\right) \\
& y_{0} \in \mathcal{N}_{\eta}\left(g_{1} P_{1}\right) \cap \mathcal{N}_{\eta}\left(g_{3} P_{3}\right) \\
& y_{n} \in \mathcal{N}_{\eta}\left(g_{3} P_{3}\right) \cap \mathcal{N}_{\eta}\left(g_{0} P_{0}\right)
\end{aligned}
$$

Now Lemma 5.7 implies that either all four cosets are equal, or some pair from the set $\left\{x_{n}, x_{0}, y_{0}, y_{n}\right\}$ has distance less than $\xi$, for some constant $\xi=\xi(\eta)$.

We can now complete the proof using arguments similar to the ones used above. As we have seen, it suffices to assume that $g_{0} P_{0} \notin\left\{g_{1} P_{1}, g_{2}, P_{2}\right\}$. Therefore some pair from $\left\{x_{n}, x_{0}, y_{0}, y_{n}\right\}$ is within a distance $\xi$. If $d\left(y_{0}, y_{n}\right)<\xi$, then the result follows from an application of Lemma 5.6. If $d\left(x_{0}, x_{n}\right)<\xi$, then $d\left(A_{n}, g_{0} P_{0}\right)<R_{0}+R_{1}+\xi$. If $d\left(\left\{x_{0}, x_{n}\right\},\left\{y_{0}, y_{n}\right\}\right)<\xi$ then, since $y_{0}, y_{n} \in A_{0}$, it follows that either $x_{0}$ or $x_{n}$ is within a distance $\xi$ of $A_{0}$ and within a distance $R_{0}+R_{1}$ of $A_{1}, \ldots, A_{n-1}$, contradicting (7-2) once $M>\xi+R_{0}+R_{1}$.

Proposition 7.8 (Close to two peripherals) Let $(G, \mathbb{P})$ be relatively hyperbolic. For each $M>0$ and $\sigma>0$ there exists $M_{1}=M_{1}(M, \sigma)$ such that the following holds. Suppose $\mathcal{A}$ is a collection of $\sigma$-relatively quasiconvex subsets and $g_{0} P_{0} \neq g_{1} P_{1}$ are distinct peripheral cosets. If $d_{\mathcal{S}}\left(g_{i} P_{i}, A\right)<M$ for all $i \in\{0,1\}$ and for all $A \in \mathcal{A}$, then the following intersection is nonempty:

$$
\bigcap_{A \in \mathcal{A}} \mathcal{N}_{M_{1}}(A)
$$

Proof Let $\epsilon, R$ and $L$ be the constants given by Proposition 6.7, let $\nu_{0}$ be the constant given by Proposition 5.4, let $v:=v_{0}+M+\epsilon$, and let $D_{1}=D_{1}(v)$ be given by Proposition 5.4. Let $\tau=\tau(v)$ be given by Theorem 5.3, and let $\kappa=\kappa(\epsilon+\tau)$ be given by Theorem 5.2 .

For each $A \in \mathcal{A}$ there exists a geodesic $c$ in $\operatorname{Cayley}(G, \mathcal{S})$ whose endpoints $x_{0}, x_{1}$ satisfy $d_{\mathcal{S}}\left(x_{i}, g_{i} P_{i}\right)<M$. Choose $y \in c$ to be the last point of $c$ that lies in $\overline{\mathcal{N}_{v}\left(g_{0} P_{0}\right)}$ (when $c$ is traversed from $x_{0}$ to $x_{1}$ ).

We will see that $y$ is within an $\mathcal{S}$-distance $\kappa$ of an $(\epsilon, R)$-transition point of $c$. Suppose $y$ is not itself an $(\epsilon, R)$-transition point. Then by Proposition 6.7 there is 
a subgeodesic $\bar{c}$ of $c$ containing $y$ each of whose interior points is $(\epsilon, R)$-deep in a fixed peripheral coset $g P$ and whose endpoints are $(\epsilon, R)$-transition points. It is clear that $g P \neq g_{0} P_{0}$, since $\epsilon \leq v$. Let $\left[x_{0}, y\right]$ denote the portion of $c$ from $x_{0}$ to $y$. Since $M \leq v$, the points $x_{0}$ and $y$ each lie in $\mathcal{N}_{v}\left(g_{0} P_{0}\right)$. Therefore, $\left[x_{0}, y\right]$ lies in $\mathcal{N}_{\tau}\left(g_{0} P_{0}\right)$. On the other hand, $\bar{c}$ lies in $\mathcal{N}_{\epsilon}(g P)$. Thus $\bar{c} \cap\left[x_{0}, y\right]$ lies in $\mathcal{N}_{\epsilon}(g P) \cap \mathcal{N}_{\tau}\left(g_{0} P_{0}\right)$ and hence has length at most $\kappa$. In other words, $y$ is within an $\mathcal{S}$-distance $\kappa$ of an $(\epsilon, R)$-transition point $z$ of $c$, as desired.

By Proposition 6.7 this point $z$ is within an $\mathcal{S}$-distance $L$ of a vertex $w$ of $\widehat{c}$, where $\widehat{c}$ is any relative geodesic in $\operatorname{Cayley}(G, \mathcal{S} \cup \mathcal{P})$ with the same endpoints as $c$. Since $A$ is $\sigma-$ relatively quasiconvex, it follows that $d_{\mathcal{S}}(w, A)<\sigma$. Therefore, $d_{\mathcal{S}}(y, A)<\kappa+L+\sigma$.

If we now vary the choice of $A$, the above argument produces for each $A \in \mathcal{A}$ a point $y=y(A)$ within an $\mathcal{S}$-distance $\kappa+L+\sigma$ of $A$. Since $y$ is the last point of $c$ that lies in $\overline{\mathcal{N}_{v}\left(g_{0} P_{0}\right)}$, it follows from Proposition 5.4 that for each $A, A^{\prime} \in \mathcal{A}$ we have $d_{\mathcal{S}}\left(y(A), y\left(A^{\prime}\right)\right)<D_{1}$. In particular, if we fix $A \in \mathcal{A}$, then every $A^{\prime} \in \mathcal{A}$ comes within an $\mathcal{S}$-distance $D_{1}+\kappa+L+\sigma$ of the point $y(A)$. Setting $M_{1} \geq D_{1}+\kappa+L+\sigma$ completes the proof.

The following corollary essentially states that, if we increase the constants given by Proposition 7.7, then the peripheral coset appearing in conclusion (2) is unique.

Corollary 7.9 (Uniqueness of peripheral coset) Let $(G, \mathbb{P})$ be relatively hyperbolic. Choose $\sigma, D, n$ and $\mathcal{A}$ satisfying the hypothesis of Proposition 7.7, and let $M_{0}=$ $M_{0}(\sigma, D, n)$ be the constant given by Proposition 7.7. Then for each $M \geq M_{0}$ there exists $M_{2}=M_{2}(\sigma, D, n, M)$ such that at least one of the following holds:

(1) For every $\left\{A_{1}, \ldots, A_{n}\right\} \subseteq \mathcal{A}$, the following intersection is nonempty:

$$
\bigcap_{\ell=1}^{n} \mathcal{N}_{M_{2}}\left(A_{\ell}\right)
$$

(2) There is a unique peripheral coset $g P$ such that $d_{\mathcal{S}}(g P, A)<M$ for all $A \in \mathcal{A}$. Furthermore, for all $A, A^{\prime} \in \mathcal{A}$ the following intersection is nonempty:

$$
\mathcal{N}_{M}(g P) \cap \mathcal{N}_{M}(A) \cap \mathcal{N}_{M}\left(A^{\prime}\right)
$$

Proof Choose $M \geq M_{0}$ arbitrary. If there does not exist a peripheral coset $g P$ such that for all $A \in \mathcal{A}$ we have $d_{\mathcal{S}}(g P, A)<M$, then Proposition 7.7 implies that (1) holds for any $M_{2} \geq M$. 
On the other hand, suppose there are two peripheral cosets $g_{0} P_{0} \neq g_{1} P_{1}$ such that $d_{\mathcal{S}}\left(g_{i} P_{i}, A\right)<M$ for all $i \in\{0,1\}$ and all $A \in \mathcal{A}$. In this case, Proposition 7.8 implies that

$$
\bigcap_{A \in \mathcal{A}} \mathcal{N}_{M_{2}}(A)
$$

is nonempty once $M_{2}$ is larger than the constant $M_{1}=M_{1}(M, \sigma)$. Condition (1) follows immediately.

\section{Bounded packing in relatively hyperbolic groups}

Let $(G, \mathbb{P})$ be relatively hyperbolic. An element $f \in G$ is elliptic if it has finite order. If $f$ has infinite order, then $f$ is parabolic if it is contained in a conjugate of a peripheral subgroup $P \in \mathbb{P}$ and loxodromic otherwise. A subgroup $H \leq G$ is elliptic if it is finite, and parabolic if it is infinite and contained in a conjugate of a peripheral subgroup $P \in \mathbb{P}$. Otherwise $H$ is loxodromic. Every loxodromic subgroup contains a loxodromic element.

Definition 8.1 Let height $_{(G, \mathbb{P})}(H)=n$ if $n$ is the maximal number of distinct cosets $g_{i} H$ such that the intersection $\bigcap g_{i} H g_{i}^{-1}$ is loxodromic. Let $\operatorname{width}_{(G, \mathbb{P})}(H)=n$ if $n$ is the maximal number of distinct cosets $g_{i} H$ such that for each $i, j$ the intersection $g_{i} H g_{i}^{-1} \cap g_{j} H g_{j}^{-1}$ is loxodromic. Note that an elliptic or parabolic subgroup has both height and width zero.

Lemma 8.2 Let $(G, \mathbb{P})$ be relatively hyperbolic, and let $\mathcal{S}$ be a finite generating set for $G$. There is a universal constant $L \geq 0$ so that the following holds. Let $c$ and $c^{\prime}$ be relative geodesic lines in $\operatorname{Cayley}(G, \mathcal{S} \cup \mathcal{P})$ with the same endpoints at infinity. Then the set of vertices of $c$ and the set of vertices of $c^{\prime}$ are within a Hausdorff distance $L$ with respect to the metric $d_{\mathcal{S}}$.

Proof Since $c$ and $c^{\prime}$ have the same endpoints at infinity, they are at a finite Hausdorff distance, say $D$, in the relative metric $d_{\mathcal{S} \cup \mathcal{P}}$. Let $v$ be the constant given by Theorem 6.2. Choose a vertex $v$ of $c$. We will show that the $\mathcal{S}$-distance from $v$ to some vertex $v^{\prime}$ of $c^{\prime}$ is bounded above by a constant that does not depend on $D$.

Let $x$ and $y$ be vertices of $c$ on either side of $v$, at an $(\mathcal{S} \cup \mathcal{P})$-distance at least $D+2 v$ from $v$. Choose vertices $x^{\prime}$ and $y^{\prime}$ of $c^{\prime}$ such that $d_{\mathcal{S} \cup \mathcal{P}}\left(x, x^{\prime}\right)<D$ and $d_{\mathcal{S} \cup \mathcal{P}}\left(y, y^{\prime}\right)<D$. Let $\bar{c}$ be the subpath of $c^{\prime}$ from $x^{\prime}$ to $y^{\prime}$, and choose relative geodesics $c_{0}$ from $x$ to $x^{\prime}$ and $c_{1}$ from $y^{\prime}$ to $y$. By Corollary 6.3, the vertex $v$ is within an $\mathcal{S}$-distance $2 v$ of a vertex $v^{\prime}$ of $c_{0} \cup \bar{c} \cup c_{1}$. However, by the triangle inequality, the $(\mathcal{S} \cup \mathcal{P})$-distance from $v$ to any vertex of $c_{0} \cup c_{1}$ is greater than $2 v$. Therefore $v^{\prime}$ is a vertex of $\bar{c}$. 
Lemma 8.3 Let $(G, \mathbb{P})$ be relatively hyperbolic with a finite generating set $\mathcal{S}$. Suppose $H \leq G$ is $\sigma$-relatively quasiconvex. If $f$ is a loxodromic element of $H$, then there exists a bi-infinite relative geodesic $c$ in $\operatorname{Cayley}(G, \mathcal{S} \cup \mathcal{P})$ such that the following hold:

(1) The Hausdorff $\mathcal{S}$-distance between the set of vertices of $c$ and the subgroup $\langle f\rangle$ is finite.

(2) Each vertex $v$ of $c$ lies in the $\sigma$-neighborhood of $H$.

Proof For each $n=1,2,3, \ldots$ choose a relative geodesic $c_{n}$ connecting $f^{-n}$ with $f^{n}$. Since $H$ is $\sigma$-relatively quasiconvex, each vertex of $c_{n}$ lies in the $\sigma$-neighborhood of $H$. To complete the proof, it suffices to show that the sequence $\left\{c_{n}\right\}$ has a subsequence that converges pointwise to a relative geodesic line $c$. In order to ensure the existence of such a subsequence we will show that the vertices of $c_{n}$ track $\mathcal{S}$-close to the set $\left\{f^{i} \mid-n \leq i \leq n\right\}$.

The subgroup $\langle f\rangle$ is $L$-relatively quasiconvex in $G$ for some $L=L(f)$, and $\langle f\rangle \cap g P g^{-1}$ is finite for all conjugates of peripheral subgroups $g P$ by Osin [15, Theorem 4.19]. Furthermore, there exists $\epsilon=\epsilon(f)$ such that the inclusion $\langle f\rangle \rightarrow$ Cayley $(G, \mathcal{S} \cup \mathcal{P})$ is an $\epsilon$-quasi-isometric embedding with respect to the word metric on $\langle f\rangle$ for the standard generating set $\{f\}$ (see Osin [15, Corollary 4.20]). Let $D$ be an upper bound on the $\mathcal{S}$-diameters of the finitely many finite subgroups $\langle f\rangle \cap g P g^{-1}$ with $|g|_{\mathcal{S}}<L$.

By quasiconvexity, each vertex of $c_{n}$ lies $\mathcal{S}$-near $\langle f\rangle$. We need to show that if $-n \leq i \leq n$ the vertex $f^{i}$ lies $\mathcal{S}$-near some vertex of $c_{n}$. Indeed, quasiconvexity guarantees that some of the $f^{i}$ lie within an $\mathcal{S}$-distance $\sigma^{\prime}$ of vertices of $c_{n}$, so we only need to bound the size of the "gaps" between these elements $f^{i}$.

Let us begin with a pair of vertices $v$ and $w$ in the $L$-neighborhood of $\langle f\rangle$ connected by an edge $e$ in Cayley $(G, \mathcal{S} \cup \mathcal{P})$. Translate by an element of $\langle f\rangle$ so that $v$ and $w$ are within an $\mathcal{S}$-distance $L$ of 1 and $f^{k}$ for some $k \geq 0$. If $e$ is labelled by a generator $s \in \mathcal{S}$ then $\left|f^{k}\right|_{\mathcal{S}}$ is at most $2 L+1$. On the other hand, suppose $e$ is labelled by a parabolic element $p \in P$ for some $P \in \mathbb{P}$. Then both endpoints of $e$ lie in one of the finitely many peripheral cosets $g P$ with $|g|_{\mathcal{S}}<L$. By Lemma 4.5 we have

$$
\left\{1, f^{k}\right\} \subseteq \mathcal{N}_{L}(\langle f\rangle) \cap \mathcal{N}_{L}(g P) \subseteq \mathcal{N}_{L^{\prime}}\left(\langle f\rangle \cap g P g^{-1}\right)
$$

for some constant $L^{\prime}$. Maximizing over the finitely many possibilities for $g P$, we can assume that $L^{\prime}$ does not depend on the choice of edge $e$. Observe that $\left|f^{k}\right|_{\mathcal{S}}<2 L^{\prime}+D$. Thus in all possible cases, we have

$$
\left|f^{k}\right|_{\mathcal{S}}<M:=\max \left\{2 L+1,2 L^{\prime}+D\right\} .
$$


It follows that:

$$
0 \leq k \leq \epsilon\left|f^{k}\right|_{\mathcal{S} \cup \mathcal{P}}+\epsilon \leq \epsilon\left|f^{k}\right|_{\mathcal{S}}+\epsilon \leq \epsilon M+\epsilon
$$

On the other hand, whenever $0 \leq i \leq k$ we have:

$$
\left|f^{i}\right|_{\mathcal{S}} \leq i|f|_{\mathcal{S}} \leq k|f|_{\mathcal{S}} \leq(\epsilon M+\epsilon)|f|_{\mathcal{S}}
$$

Therefore the $\mathcal{S}$-distance from $f^{i}$ to $\{v, w\}$ is bounded above by the constant:

$$
R:=L+(\epsilon M+\epsilon)|f|_{\mathcal{S}}
$$

By quasiconvexity, each vertex of $c_{n}$ lies within an $\mathcal{S}$-distance $L$ of some element of $\langle f\rangle$. Since $c_{n}$ is an edge path, we can apply the above argument to the set of vertices of the path $c_{n}$ to see that whenever $-n \leq i \leq n$ the vertex $f^{i}$ lies within an $\mathcal{S}$-distance $R$ of some vertex of $c_{n}$. Since $L \leq R$, the Hausdorff distance between the vertices of $c_{n}$ and the set $\left\{f^{i} \mid-n \leq i \leq n\right\}$ is at most $R$. An easy diagonal argument shows that the sequence of relative geodesics $\left\{c_{n}\right\}$ has a subsequence converging pointwise to a relative geodesic line $c$.

Clearly the Hausdorff $\mathcal{S}$-distance between the vertices of $c$ and the subgroup $\langle f\rangle$ is at most $R$. Since the vertices of $c_{n}$ lie in the $\sigma$-neighborhood of $H$, the same holds for the vertices of $c$, completing the proof of the lemma.

Lemma 8.4 (Loxodromic virtual intersection implies close) Let $(G, \mathbb{P})$ be relatively hyperbolic, let $\mathcal{S}$ be a finite generating set for $G$, and choose $\sigma \geq 0$. There is a constant $C$ so that the following holds. Let $H$ be a $\sigma$-relatively quasiconvex subgroup of $(G, \mathbb{P})$ containing a loxodromic element $f$. Suppose $\mathcal{A}=\left\{g_{i} H \mid i \in I\right\}$ is a collection of distinct cosets of $H$ such that each conjugate $\mathrm{g}_{i} \mathrm{Hg}_{i}^{-1}$ contains some power $f^{k_{i}}$ of $f$. Then there exists a vertex $z \in G$ such that the ball of radius $C$ in Cayley $(G, \mathcal{S})$ intersects every coset $g_{i} H \in \mathcal{A}$.

Proof By Osin [15, Corollary 4.20], the map $n \mapsto f^{n}$ is a quasigeodesic line in Cayley $(G, \mathcal{S} \cup \mathcal{P})$. Therefore the set $\left\{f^{n} \mid n \in \mathbb{Z}\right\}$ has exactly two limit points $f^{ \pm \infty}$ in $\partial$ Cayley $(G, \mathcal{S} \cup \mathcal{P})$. If $g_{i} H \in \mathcal{A}$ then $f^{k_{i}} \in g_{i} H g_{i}^{-1}$ for some $k_{i} \in \mathbb{Z}$. Thus $f^{n k_{i}} g_{i} \in g_{i} H$ for all $n \in \mathbb{Z}$. The sets $\left\{f^{n k_{i}} g_{i} \mid n \in \mathbb{Z}\right\}$ and $\left\{f^{n} \mid n \in \mathbb{Z}\right\}$ are at a finite Hausdorff $\mathcal{S}$-distance and thus have the same limit points $f^{ \pm \infty}$.

Translating $g_{i} H$ by $g_{i}^{-1}$, we see that the subgroup $\left\langle g_{i}^{-1} f^{k_{i}} g_{i}\right\rangle$ of $H$ has limit points $g_{i}^{-1} f^{ \pm \infty}$. By Lemma 8.3 the points $g_{i}^{-1} f^{ \pm \infty}$ can be connected by a relative geodesic line $c_{i}$ such that each vertex of $c_{i}$ lies within an $\mathcal{S}$-distance $\sigma$ of $H$. Translating back by $g_{i}$ produces a relative geodesic line $g_{i} c_{i}$ with endpoints $f^{ \pm \infty}$ such that each vertex of $g_{i} c_{i}$ lies in the $\sigma$-neighborhood of $g_{i} H$. 
For each $i, j \in I$ the relative geodesics $g_{i} c_{i}$ and $g_{j} c_{j}$ have the same endpoints at infinity. By Lemma 8.2 the Hausdorff $\mathcal{S}$-distance between the vertex sets of $g_{i} c_{i}$ and $g_{j} c_{j}$ is at most $L$. Therefore for each vertex $z$ of $g_{i} c_{i}$, the coset $g_{j} H$ intersects the ball of radius $L+\sigma$ in Cayley $(G, \mathcal{S})$ centered at $z$.

The following special case of Lemma 8.4 is the main connection between height/width and packing. Roughly speaking, a collection of conjugates with loxodromic intersection comes mutually close together in $\operatorname{Cayley}(G, \mathcal{S})$.

Corollary 8.5 (Loxodromic intersection implies close) Let $(G, \mathbb{P})$ be relatively hyperbolic, let $\mathcal{S}$ be a finite generating set for $G$, and choose $\sigma \geq 0$. There is a constant $C$ so that the following holds. Let $H$ be a $\sigma$-relatively quasiconvex subgroup of $(G, \mathbb{P})$. Suppose $\left\{g_{i} H \mid i \in I\right\}$ is a collection of distinct cosets of $H$ such that $\bigcap g_{i} \mathrm{Hg}_{i}^{-1}$ is loxodromic. Then there exists a vertex $z \in G$ such that the ball of radius $C$ in Cayley $(G, \mathcal{S})$ intersects every coset $g_{i} H$.

The following corollary is an immediate consequence of the preceding result together with the local finiteness of Cayley $(G, \mathcal{S})$.

Corollary 8.6 Let $(G, \mathbb{P})$ be relatively hyperbolic and let $H \leq G$ be relatively quasiconvex. Then height $(G, \mathbb{P})(H)$ is finite.

Corollary 8.5 also has the following easy consequence.

Corollary 8.7 Let $(G, \mathbb{P})$ be relatively hyperbolic and let $H \leq G$ be a relatively quasiconvex subgroup. There are only finitely many double cosets $\mathrm{Hg}_{1} \mathrm{H}, \ldots, \mathrm{Hg}_{n} \mathrm{H}$ such that $H \cap g_{i} \mathrm{Hg}_{i}^{-1}$ is loxodromic.

Proof Suppose $\left\{g_{i} H \mid i \in I\right\}$ is a collection of cosets such that $H \cap g_{i} H_{i}^{-1}$ is loxodromic for each $i$. By Corollary 8.5, the distances $d_{\mathcal{S}}\left(H, g_{i} H\right)$ are bounded above by a constant $C$. Thus we can translate $g_{i} H$ by an element of $H$ to obtain a coset $h g_{i} H$ intersecting the ball of radius $C$ in $\operatorname{Cayley}(G, \mathcal{S})$ centered at the identity. Since this ball is finite, it follows that the cosets $g_{i} H$ lie in only finitely many double cosets $H g_{i} H$.

Theorem 1.6 also follows from Lemma 8.4.

Proof of Theorem 1.6 Let $\mathcal{A}=\left\{g_{i} H \mid i \in I\right\}$ be the set of all cosets $g_{i} H$ such that $g_{i} H g_{i}^{-1} \cap H$ has finite index in both $g_{i} H g_{i}^{-1}$ and $H$. Then the union of all cosets in $\mathcal{A}$ is the commensurator of $H$ in $G$, and the cardinality of $\mathcal{A}$ is equal to the index of $H$ 
in the commensurator. Since $H$ is loxodromic, it contains a loxodromic element $f$. If $g_{i} H \in \mathcal{A}$ then $f^{k_{i}} \in g_{i} H g_{i}^{-1}$ for some $k_{i} \in \mathbb{Z}$. Thus by Lemma 8.4 there is a vertex $z$ such that the ball of radius $C$ in Cayley $(G, \mathcal{S})$ centered at $z$ intersects each coset $g_{i} H \in \mathcal{A}$. The finite cardinality of this ball is an upper bound for the size of $\mathcal{A}$.

Proposition 8.8 (Packing peripheral subgroups) If $(G, \mathbb{P})$ is relatively hyperbolic then each peripheral subgroup $P \in \mathbb{P}$ has bounded packing in $G$.

Proof Choose a finite generating set $\mathcal{S}$ for $G$. Let $\mathcal{A}$ be a collection of left cosets of $P$ whose pairwise distances are less than $D$. Consider three distinct elements $g_{0} P$, $g_{1} P, g_{2} P$ of $\mathcal{A}$. Choose points $x_{0} \in \mathcal{N}_{D}\left(g_{1} P\right) \cap \mathcal{N}_{D}\left(g_{2} P\right)$ and $x_{1} \in \mathcal{N}_{D}\left(g_{0} P\right) \cap$ $\mathcal{N}_{D}\left(g_{2} P\right)$. Then by Lemma 5.6, there is a constant $\zeta=\zeta(D)$ such that $d\left(x_{0}, x_{1}\right)<\zeta$. Therefore $g_{0} P$ intersects the ball of radius $\zeta+D$ centered at $x_{0}$. Keeping $g_{1} P$ and $g_{2} P$ fixed and varying $g_{0} P$ over all elements of $\mathcal{A}-\left\{g_{1} P, g_{2} P\right\}$, we see that every element of $\mathcal{A}$ intersects this ball. Since the metric $d_{\mathcal{S}}$ is proper and left invariant, the size of $\mathcal{A}$ is bounded in terms of $D$.

Theorem 8.9 (Packing relative to peripheral subgroups) Let $(G, \mathbb{P})$ be relatively hyperbolic, choose a finite generating set $\mathcal{S}$ for $G$, and let $\mathcal{H}$ be a finite collection of $\sigma$-relatively quasiconvex subgroups of $G$. For each constant $D$, there are constants $R$ and $M_{3}$ such that the following holds. Let $\mathcal{A}$ be any set of left cosets $g H$ with $g \in G$ and $H \in \mathcal{H}$ such that for all $g H, g^{\prime} H^{\prime} \in \mathcal{A}$ we have $d_{\mathcal{S}}\left(g H, g^{\prime} H^{\prime}\right)<D$. Suppose the following intersection is empty:

$$
\bigcap_{g H \in \mathcal{A}} \mathcal{N}_{M_{3}}(g H)=\varnothing
$$

Then we have the following:

(1) There is a unique peripheral coset $g P$ such that for all $a H \in \mathcal{A}$ the intersection $\mathcal{N}_{R}(g P) \cap \mathcal{N}_{R}(a H)$ is nonempty.

(2) $\mathcal{N}_{R}(g P) \cap \mathcal{N}_{R}(a H) \cap \mathcal{N}_{R}\left(a^{\prime} H^{\prime}\right)$ is nonempty for all $a H, a^{\prime} H^{\prime} \in \mathcal{A}$.

(3) $\mathcal{N}_{R}(g P) \cap \mathcal{N}_{R}(a H)$ is unbounded for all $a H \in \mathcal{A}$.

(4) $g P g^{-1} \cap a \mathrm{Ha}^{-1}$ is infinite for all $a H \in \mathcal{A}$.

Proof We will first prove assertions (1) and (2). Let

$$
n=1+\sum_{H \in \mathcal{H}} \operatorname{height}_{(G, \mathbb{P})}(H),
$$

which is known to be finite by Corollary 8.6. Let $M_{0}=M_{0}(\sigma, D, n+1)$ be the constant given by Proposition 7.7, and let $M_{2}=M_{2}\left(\sigma, D, n+1, M_{0}\right)$ be the constant given by 
Corollary 7.9. If there exist $n+1$ elements of $\mathcal{A}$ whose $M_{2}$-neighborhoods have empty intersection, then Corollary 7.9 gives (1) and (2) immediately, using $R=M_{0}$. Thus it suffices to assume that for all $g_{1} H_{1}, \ldots, g_{n+1} H_{n+1} \in \mathcal{A}$ the following intersection is nonempty:

$$
\mathcal{N}_{M_{2}}\left(g_{1} H_{1}\right) \cap \cdots \cap \mathcal{N}_{M_{2}}\left(g_{n+1} H_{n+1}\right)
$$

Fix $n$ distinct cosets $g_{1} H_{1}, \ldots, g_{n} H_{n} \in \mathcal{A}$. (If we choose $M_{3} \geq M_{2}$, then (8-1) implies that $\mathcal{A}$ has at least $n+2$ elements.) Consider the following intersection of conjugates:

$$
K:=g_{1} H_{1} g_{1}^{-1} \cap \cdots \cap g_{n} H_{n} g_{n}^{-1}
$$

By our choice of $n$, some $H \in \mathcal{H}$ appears at least $1+$ height $(H)$ times in the list $H_{1}, \ldots, H_{n}$. Thus $K$ is contained in the intersection of at least $1+\operatorname{height}(H)$ distinct conjugates of $H$, so that $K$ is either elliptic or parabolic.

Case 1 Suppose $K$ is elliptic. Once $M_{3}$ is chosen sufficiently large, we will reach a contradiction with (8-1). Translating $\mathcal{A}$ by an element of $G$, we can assume, without loss of generality, that the cosets $g_{1} H_{1}, \ldots, g_{n} H_{n}$ all intersect the ball of radius $M_{2}$ centered at the identity. Since there are only finitely many possibilities for these cosets, our choice of $M_{3}$ is permitted to depend on $g_{1} H_{1}, \ldots, g_{n} H_{n}$. (Maximizing over all such cosets would give a uniform constant $M_{3}$.)

By Corollary 4.6 we have

$$
\mathcal{N}_{M_{2}}\left(g_{1} H_{1}\right) \cap \cdots \cap \mathcal{N}_{M_{2}}\left(g_{n} H_{n}\right) \subseteq \mathcal{N}_{M^{\prime}}(K)
$$

for some $M^{\prime}$ depending on $M_{2}$ and the cosets $g_{1} H_{1}, \ldots, g_{n} H_{n}$. If $a H$ is any left coset in $\mathcal{A}$, the following intersection is nonempty by hypothesis:

$$
\mathcal{N}_{\boldsymbol{M}_{2}}(a H) \cap\left(\mathcal{N}_{\boldsymbol{M}_{2}}\left(g_{1} H_{1}\right) \cap \cdots \cap \mathcal{N}_{\boldsymbol{M}_{2}}\left(g_{n} H_{n}\right)\right) .
$$

Thus by (8-2) we have $d_{\mathcal{S}}(a H, K)<M_{2}+M^{\prime}$. Since $K$ is an elliptic subgroup, it has a finite diameter $D=D(K)$ and contains the identity. Hence every $a H \in \mathcal{A}$ intersects the finite ball of radius $D+M_{2}+M^{\prime}$ centered at the identity, which contradicts (8-1) if we choose $M_{3} \geq D+M_{2}+M^{\prime}$.

Case 2 Suppose $K$ is parabolic. By Proposition 7.4, for each $H \in \mathcal{S}$ and each peripheral subgroup $P$, the peripheral cosets $g P$ such that $H \cap g P g^{-1}$ is infinite lie in finitely many $H$-orbits. Let $T$ be the maximum distance between $H$ and any coset $g P$ such that $H \cap g P g^{-1}$ is infinite. Equivalently, if $\mathrm{gHg}^{-1} \cap P$ is infinite, then the distance between $g H$ and $P$ is at most $T$.

Since $K$ is parabolic, it is contained in a conjugate of some peripheral subgroup $P$. Translating $\mathcal{A}$ by an element of $G$, we can assume that $K$ is a subgroup of $P$ itself. 
For each $i=1, \ldots, n$ the group $g_{i} H_{i} g_{i}^{-1} \cap P$ is infinite, since it contains the infinite group $K$. Therefore the distance between $g_{i} H_{i}$ and $P$ is at most $T$. By assumption, the following intersection is nonempty:

$$
\mathcal{N}_{M_{2}}\left(g_{1} H_{1}\right) \cap \cdots \cap \mathcal{N}_{M_{2}}\left(g_{n} H_{n}\right)
$$

Thus by Corollary 7.6 there is a constant $R_{1}=R_{1}\left(\sigma, T+M_{2}, n\right)$ and a point

$$
x \in \mathcal{N}_{R_{1}}(P) \cap \mathcal{N}_{R_{1}}\left(g_{1} H_{1}\right) \cap \cdots \cap \mathcal{N}_{R_{1}}\left(g_{n} H_{n}\right) .
$$

Translating $\mathcal{A}$ by an element of $P$ we can assume without loss of generality that $x$ lies in the ball of radius $R_{1}$ centered at the identity. Consequently, the cosets $g_{1} H_{1}, \ldots, g_{n} H_{n}$ each intersect the ball of radius $2 R_{1}$ centered at the identity. As in the elliptic case, there are a uniformly bounded number of choices for $g_{1} H_{1}, \ldots, g_{n} H_{n}$.

As before, (8-2) holds for some $M^{\prime}$ depending on $g_{1} H_{1}, \ldots, g_{n} H_{n}$ and $M_{2}$. Since $K \leq P$, it follows from the same reasoning as above that $d_{\mathcal{S}}(a H, P) \leq d_{\mathcal{S}}(a H, K)<$ $M_{2}+M^{\prime}$ for each $a H \in \mathcal{A}$. Furthermore, for each $a H, a^{\prime} H^{\prime} \in \mathcal{A}$, the pairwise distances between $a H, a^{\prime} H^{\prime}$ and $P$ are at most $D+M_{2}+M^{\prime}$. Thus Proposition 7.5(2) gives a constant $R=R\left(\sigma, D+M_{2}+M^{\prime}\right)$ such that for all $a H, a^{\prime} H^{\prime} \in \mathcal{A}$ the following intersection is nonempty:

$$
\mathcal{N}_{R}(P) \cap \mathcal{N}_{R}(a H) \cap \mathcal{N}_{R}\left(a^{\prime} H^{\prime}\right)
$$

Recall that $R$ depends on the cosets $g_{1} H_{1}, \ldots, g_{n} H_{n}$, which each intersect the ball of radius $2 R_{1}$ centered at the identity. Maximizing over the finitely many possible choices for these cosets gives a uniform constant-which we again call $R$ - that does not depend on our particular choice of $\mathcal{A}$.

In order to prove (1) and (2) it suffices to establish the uniqueness of $P$ in the sense of (1) once $M_{3}$ is chosen sufficiently large. Suppose by way of contradiction that there were another peripheral coset $g^{\prime} P^{\prime} \neq P$ such that for all $a H \in \mathcal{A}$ the intersection $\mathcal{N}_{R}\left(g^{\prime} P^{\prime}\right) \cap \mathcal{N}_{R}(a H)$ is nonempty. Then Proposition 7.8 implies that the intersection $\bigcap_{a H \in \mathcal{A}} \mathcal{N}_{M_{1}}(a H)$ is nonempty for $M_{1}=M_{1}(R, \sigma)$. If we choose $M_{3}$ larger than $M_{1}$, this clearly contradicts (8-1).

We will now show that (2) implies (3) when $M_{3}$ is chosen sufficiently large. Indeed, suppose by way of contradiction that there exists $a H \in \mathcal{A}$ such that $\mathcal{N}_{R}(g P) \cap \mathcal{N}_{R}(a H)$ is bounded. After translating $\mathcal{A}$ by an element of $G$, we can assume that $g P$ and $a H$ both intersect the ball of radius $R$ in $G$. Thus there are a bounded number of choices for the cosets $g P$ and $a H$, up to translation in $G$. Maximizing over all such choices gives a uniform upper bound $S$ on the diameter of the intersection $I:=\mathcal{N}_{R}(g P) \cap \mathcal{N}_{R}(a H)$. If $a^{\prime} H^{\prime}$ is any other element of $\mathcal{A}$, then (2) implies that $\mathcal{N}_{R}\left(a^{\prime} H^{\prime}\right)$ intersects $I$. 
Therefore every $a^{\prime} H^{\prime} \in \mathcal{A}$ intersects a fixed set of diameter $S+R$, which contradicts (8-1) if we choose $M_{3}$ larger than $S+R$.

Finally observe that (4) follows immediately from (3) using Lemma 4.5.

Theorem 8.10 (Packing relatively quasiconvex subgroups) Let $(G, \mathbb{P})$ be relatively hyperbolic, choose a finite generating set $\mathcal{S}$ for $G$, and let $H$ be a $\sigma$-relatively quasiconvex subgroup of $G$. Suppose for each peripheral subgroup $P \in \mathbb{P}$ and each $g \in G$ the intersection $P \cap g \mathrm{Hg}^{-1}$ has bounded packing in $P$. Then $H$ has bounded packing in $G$.

Proof Let $\mathcal{A}$ be any set of left cosets $g H$ whose pairwise distances are at most $D$. Our goal is to show that $\mathcal{A}$ is finite and to bound the cardinality of $\mathcal{A}$ as a function of $D$.

Let $R$ and $M_{3}$ be the constants given by Theorem 8.9. Suppose the following intersection is nonempty:

$$
\bigcap_{g H \in \mathcal{A}} \mathcal{N}_{M_{3}}(g H)
$$

Then there is a fixed ball of radius $M_{3}$ in $G$ that intersects every $g H \in \mathcal{A}$. The cardinality of such a ball is an upper bound on the cardinality of $\mathcal{A}$.

Thus it suffices to assume that the following intersection is empty:

$$
\bigcap_{g H \in \mathcal{A}} \mathcal{N}_{M_{3}}(g H)
$$

In this case, Theorem 8.9 applies. After translating $\mathcal{A}$ by an element of $G$, there exists a peripheral subgroup $P$ such that for all $g H, g^{\prime} H \in \mathcal{A}$ the following intersection is nonempty:

$$
\mathcal{N}_{R}(P) \cap \mathcal{N}_{R}(g H) \cap \mathcal{N}_{R}\left(g^{\prime} H\right)
$$

For each $g H \in \mathcal{A}$, there is a point $p \in P$ such that $d_{\mathcal{S}}(p, g H)<2 R$. Translating by $p^{-1}$, we see that the coset $p^{-1} g H$ intersects the ball of radius $2 R$ centered at the identity. Therefore every coset in $\mathcal{A}$ can be expressed as $p g H$ for some $p \in P$ and $|g|_{\mathcal{S}}<2 R$. The cosets $g H \in \mathcal{A}$ lie in a bounded number of orbits under the action of $P$; therefore it is enough to bound the number of elements of $\mathcal{A}$ lying in a single orbit $P(g H)$; in other words, a double coset.

Setting $Q:=P \cap g H g^{-1}$ the map $p g H \mapsto p Q$ that takes a left coset of $H$ in $P(g H)$ to the corresponding coset of $Q$ in $P$ is well-defined and injective; for $p g H=p^{\prime} g H$ if and only if $p^{-1} p^{\prime} \in P \cap g \mathrm{Hg}^{-1}=Q$ if and only if $p Q=p^{\prime} Q$. By Lemma 4.5, we have

$$
\mathcal{N}_{R}(P) \cap \mathcal{N}_{R}(g H) \subseteq \mathcal{N}_{M^{\prime}}(Q)
$$


for some $M^{\prime}$ depending on $P, g H$ and $R$. Translating by $p$ gives

$$
\mathcal{N}_{R}(P) \cap \mathcal{N}_{R}(p g H) \subseteq \mathcal{N}_{M^{\prime}}(p Q)
$$

for each $p \in P$, as illustrated in Figure 2 .

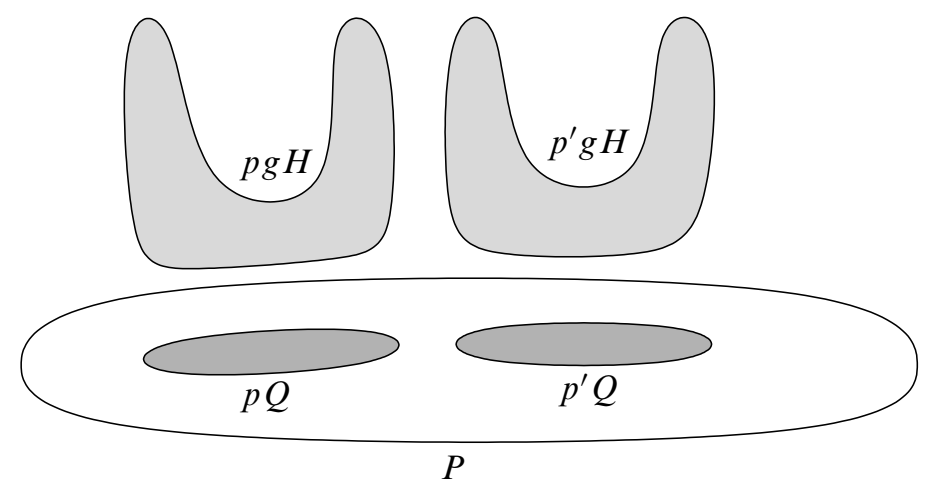

Figure 2: The coset $p Q$ is coarsely the intersection of neighborhoods of $P$ and $p g H$. The existence of a point close to $P, p g H$ and $p^{\prime} g H$ implies that $p Q$ and $p^{\prime} Q$ are close.

By hypothesis, for each $p g H, p^{\prime} g H \in \mathcal{A}$ we have

$$
\begin{aligned}
& \mathcal{N}_{R}(P) \cap \mathcal{N}_{R}(p g H) \cap \mathcal{N}_{R}\left(p^{\prime} g H\right) \\
& =\left(\mathcal{N}_{R}(P) \cap \mathcal{N}_{R}(p g H)\right) \cap\left(\mathcal{N}_{R}(P) \cap \mathcal{N}_{R}\left(p^{\prime} g H\right)\right) \neq \varnothing . \\
& \mathcal{N}_{M^{\prime}}(p Q) \cap \mathcal{N}_{M^{\prime}}\left(p^{\prime} Q\right) \neq \varnothing .
\end{aligned}
$$

But $Q=P \cap g \mathrm{Hg}^{-1}$ has bounded packing in $P$ so we have an upper bound on the number of cosets $p Q$ in $P$ that correspond to cosets $p g H \in \mathcal{A}$.

Corollary 8.11 Let $(G, \mathbb{P})$ be relatively hyperbolic and $H \leq G$ a relatively quasiconvex subgroup. Suppose $P \cap g \mathrm{Hg}^{-1}$ has bounded packing in $P$ for each peripheral subgroup $P \in \mathbb{P}$ and each $g \in G$. Then $\operatorname{width}_{(G, \mathbb{P})}(H)$ is finite.

Proof If $g_{0} H$ and $g_{1} H$ are distinct cosets such that $g_{0} H_{0}^{-1} \cap g_{1} H_{1}^{-1}$ is loxodromic, then Corollary 8.5 implies that $d_{\mathcal{S}}\left(g_{0} H, g_{1} H\right)<2 C$. The result follows immediately since $H$ has bounded packing in $G$ by Theorem 8.10. 


\section{Quasiconvex and separable implies virtually malnormal}

In this section we present a malnormality consequence of separability and finite height. The results are new even in the word-hyperbolic case.

Definition 9.1 A subgroup $H$ of $G$ is malnormal if $H \cap \mathrm{gHg}^{-1}$ is trivial for each $g \in G-H$. In applications of malnormality it is usually sufficient to know that the intersection of conjugates is finite instead of trivial, and so we have elsewhere defined $H$ to be almost malnormal if $H \cap g \mathrm{Hg}^{-1}$ is finite for each $g \in G-H$.

In light of our earlier definitions of width and height of subgroups of a relatively hyperbolic group $G$, it is natural to define a subgroup $H$ of $G$ to be relatively malnormal if $H \cap \mathrm{gHg}^{-1}$ is either elliptic or parabolic for each $g \in G-H$.

Note that any malnormal subgroup and any parabolic or elliptic subgroup is immediately relatively malnormal.

Definition 9.2 A subgroup $H$ of a group $G$ is separable if $H$ is an intersection of finite index subgroups of $G$.

Theorem 9.3 Let $H$ be a separable, relatively quasiconvex subgroup of the relatively hyperbolic group $G$. Then there is a finite index subgroup $K$ of $G$ containing $H$ such that $H$ is relatively malnormal in $K$.

Proof By Corollary 8.7, there are only finitely many double cosets $H g_{1} H, \ldots, H g_{n} H$ such that $H \cap g_{i} H_{i}^{-1}$ is loxodromic. Since $H$ is separable, there exists a finite index subgroup $K$ of $G$ such that $H \leq K$ but $g_{i} \notin K$ for $1 \leq i \leq n$. If $k \in K-H$ and $H \cap k H k^{-1}$ is loxodromic, then $k H=h g_{i} H$ for some $g_{i}$ and some $h \in H$. Therefore $h g_{i} H \subset K$ and $H \leq K$ so $g_{i} \in K$, contradicting our choice of $K$. Consequently $H$ is relatively malnormal in $K$.

Problem 9.4 Find a relatively hyperbolic group $G$ with a relatively malnormal subgroup $M$ that is not quasiconvex.

There is a related long-standing problem of constructing a malnormal subgroup of a word-hyperbolic group that is not quasiconvex. One expects that Problem 9.4 won't be much easier, even if we relax the condition of malnormality to merely require finite width. 


\section{References}

[1] J M Alonso, T Brady, D Cooper, V Ferlini, M Lustig, M Mihalik, H Short (editor), Notes on word hyperbolic groups, from: "Group theory from a geometrical viewpoint (Trieste, 1990)", (E Ghys, A Haefliger, A Verjovsky, editors), World Sci. Publ., River Edge, NJ (1991) 3-63 MR1170363

[2] H-J Bandelt, M van de Vel, Superextensions and the depth of median graphs, J. Combin. Theory Ser. A 57 (1991) 187-202 MR1111556

[3] B Bowditch, Relatively hyperbolic groups, Univ. of Southampton Preprint Ser. (1999)Http://www.warwick.ac.uk/ masgak/papers/bhb-relhyp.pdf

[4] V Chepoi, Graphs of some CAT(0) complexes, Adv. in Appl. Math. 24 (2000) 125-179 MR1748966

[5] C Druțu, M Sapir, Tree-graded spaces and asymptotic cones of groups, Topology 44 (2005) 959-1058 MR2153979 With an appendix by D Osin and Sapir

[6] B Farb, Relatively hyperbolic groups, Geom. Funct. Anal. 8 (1998) 810-840 MR1650094

[7] V N Gerasimov, Semi-splittings of groups and actions on cubings, from: "Algebra, geometry, analysis and mathematical physics (Novosibirsk, 1996)", (Y G Reshetnyak, L A Bokut', S K Vodop' yanov, I A Taĭmanov, editors), Izdat. Ross. Akad. Nauk Sib. Otd. Inst. Mat., Novosibirsk (1997) 91-109, 190 MR1624115 In Russian; translated in Siberian Adv. Math. 8 (1998) 36-58

[8] R Gitik, M Mitra, E Rips, M Sageev, Widths of subgroups, Trans. Amer. Math. Soc. 350 (1998) 321-329 MR1389776

[9] M Gromov, Hyperbolic groups, from: "Essays in group theory", (S M Gersten, editor), Math. Sci. Res. Inst. Publ. 8, Springer, New York (1987) 75-263 MR919829

[10] G C Hruska, Relative hyperbolicity and relative quasiconvexity for countable groups arXiv:0801.4596v1

[11] G C Hruska, D Wise, Finiteness properties of cubulated groups, in preparation

[12] E Martínez-Pedroza, Combination of quasiconvex subgroups of relatively hyperbolic groups, Groups Geom. Dyn. 3 (2009) 317-342

[13] G A Niblo, L D Reeves, Coxeter groups act on CAT(0) cube complexes, J. Group Theory 6 (2003) 399-413 MR1983376

[14] G A Niblo, MA Roller, Groups acting on cubes and Kazhdan's property (T), Proc. Amer. Math. Soc. 126 (1998) 693-699 MR1459140

[15] D V Osin, Relatively hyperbolic groups: intrinsic geometry, algebraic properties, and algorithmic problems, Mem. Amer. Math. Soc. 179 (2006) vi+100 MR2182268

[16] E Rips, Subgroups of small cancellation groups, Bull. London Math. Soc. 14 (1982) 45-47 MR642423 
[17] M Roller, Poc sets, median algebras and group actions. An extended study of Dunwoody's construction and Sageev's theorem, Univ. of Southampton Preprint Ser. (1998)

[18] J H Rubinstein, M Sageev, Intersection patterns of essential surfaces in 3-manifolds, Topology 38 (1999) 1281-1291 MR1690158

[19] J H Rubinstein, S Wang, $\pi_{1}$-injective surfaces in graph manifolds, Comment. Math. Helv. 73 (1998) 499-515 MR1639876

[20] M Sageev, Ends of group pairs and non-positively curved cube complexes, Proc. London Math. Soc. (3) 71 (1995) 585-617 MR1347406

[21] M Sageev, Codimension-1 subgroups and splittings of groups, J. Algebra 189 (1997) 377-389 MR1438181

Department of Mathematical Sciences, University of Wisconsin-Milwaukee PO Box 413, Milwaukee, WI 53201, USA Department of Mathematics and Statistics, McGill University Montreal, Quebec H3A 2K6, Canada chruska@uwm.edu, wise@math.mcgill.ca http://www.uwm.edu/ chruska, http://www.math.mcgill.ca/ wise

Proposed: Martin Bridson

Seconded: Walter Neumann, Benson Farb
Received: 11 September 2006 Revised: 24 March 2009 